\title{
Conservative and entropy controlled remap for multi-material ALE simulations with space-staggered schemes
}

\author{
Alexis Marbœuf ${ }^{\mathrm{b}, \mathrm{a}}$, Alexandra Claisse ${ }^{\mathrm{b}}$, Patrick Le Tallec ${ }^{\mathrm{a}, *}$ \\ ${ }^{a}$ École Polytechnique, CNRS, Université Paris-Saclay, F-91128 Palaiseau Cedex, France \\ ${ }^{b}$ CEA, DAM, DIF, F-91297 Arpajon Cedex, France
}

\begin{abstract}
The remapping strategy is crucial in any Arbitrary Lagrangian-Eulerian (ALE) algorithm based on a Lagrange-plusremap paradigm. This step is particularly challenging for space-staggered schemes since inconsistencies may appear between cell centered and node centered fields after remap if no special care is taken [1-3]. We propose here a space-staggered remapping strategy focusing on conservation properties and entropy control. The proposed algorithm conserves mass, total energy and respects the Second Law of Thermodynamics (for robustness) up to round-off errors. This is achieved at a low computational cost by introducing a consistent, explicit and local post processing of the linear momentum after remap. This new method is then analyzed showing that the strict momentum conservation is sacrificed. It is now conserved to the scheme's order, such as entropy. Other classical properties such that the "DeBar consistency" [4], the continuity with the Lagrangian step and the monotonicity are also discussed. This work is developed in the context of the intersection-based (or overlay-based) remap. Therefore, the rezoned mesh does not have to be close to the Lagrangian one and, even if it is not considered here, our study can be easily extended to rezoning strategies which modify the mesh connectivity.
\end{abstract}

Keywords: Hydrodynamics, Multi-Material ALE Schemes, Staggered Schemes, Staggered Remap, Energy Conservation, Entropy Control, Geometric Consistency

\section{Introduction}

\subsection{General framework}

For many multi-material problems such as fluid-structure interaction, impact or implosion problems, materials are in very large strains due to their nature or to the applied forces. In our situations of interest, we also have a strong coupling between energy and momentum conservation laws, due to intense transfers between internal and kinetic energies and to strong advection effects. Such situations are classically governed by the Euler's equations, written below in Lagrangian form and using a multi-material, single velocity framework

$$
\begin{cases}\frac{d \boldsymbol{x}}{d t}=\boldsymbol{u}, & \text { geometric conservation, } \\ \frac{d \rho^{\varphi}}{d t}=-\rho^{\varphi} \boldsymbol{\nabla} \cdot \boldsymbol{u}, & \text { mass conservation, } \\ \rho \frac{d \boldsymbol{u}}{d t}=-\boldsymbol{\nabla} p+\boldsymbol{\nabla} \cdot(J \tau), & \text { linear momentum, } \\ \rho^{\varphi} \frac{d e^{\varphi}}{d t}=-p^{\varphi} \boldsymbol{\nabla} \cdot \boldsymbol{u}+J \tau^{\varphi}: \boldsymbol{\nabla} \boldsymbol{u}, & \text { internal energy evolution. }\end{cases}
$$

Materials are denoted by $\varphi$ with $\boldsymbol{u}$ the velocity, $\rho^{\varphi}$ the material density, $p^{\varphi}$ the material pressure, $p$ the average pressure and $e^{\varphi}$ the material specific internal energy. The above formulation emphasizes the internal energy evolution as compared to the total energy conservation in order to focus on entropy variation. It neglects any heat exchange between neighboring points, which is justified for very fast evolutions. The constitutive materials are supposed to be

${ }^{*}$ Corresponding author.

Email address: patrick. letallec@polytechnique.edu (Patrick Le Tallec) 
non-miscible and to stay in a hydrodynamic regime, characterized by possibly very large material pressures $p^{\varphi}$ and by moderate deviatoric stress tensors $J \tau^{\varphi}$. For simplicity, it is often assumed that the internal energy is only a function $e^{\varphi}=e^{\varphi}\left(v^{\varphi}, s^{\varphi}\right)$ of the material specific volume $v^{\varphi}=\frac{1}{\rho^{\varphi}}$ (or equivalently of the transformation Jacobian $J$ since we have $\left.\frac{1}{\rho^{\varphi}}=\frac{J}{\rho_{0}^{\varphi}}\right)$ and of the material specific entropy $s^{\varphi}$. Material hydrodynamic pressure $p^{\varphi}$ and material temperature $T^{\varphi}$ are then given by

$$
\begin{gathered}
p^{\varphi}=-\left.\frac{\partial e^{\varphi}}{\partial \nu^{\varphi}}\right|_{s^{\varphi}}, \\
T^{\varphi}=\left.\frac{\partial e^{\varphi}}{\partial s^{\varphi}}\right|_{\nu^{\varphi}} .
\end{gathered}
$$

For isotropic materials in such regimes, the constitutive law characterizing the deviatoric Piola stress tensor $\tau^{\varphi}$ can be written under a simple differential form $[5,6]$

$$
\frac{d \tau^{\varphi}}{d t}=f\left(\tau^{\varphi}, \nabla \boldsymbol{u}, \ldots\right)
$$

We introduce a specific internal energy and an entropy associated to each material $\varphi$, together with the characteristic function $\mathbb{1}^{\varphi}(\boldsymbol{x})$ with value 1 if the point $\boldsymbol{x}$ is part of material $\varphi$ and 0 otherwise. Then, "average" quantities can be defined by

$$
\rho=\sum_{\varphi} \mathbb{1}^{\varphi} \rho^{\varphi}, \quad p=\sum_{\varphi} \mathbb{1}^{\varphi} p^{\varphi}, \quad \tau=\sum_{\varphi} \mathbb{1}^{\varphi} \tau^{\varphi} .
$$

Arbitrary-Lagrangian-Eulerian (ALE) techniques [7] are required in this context to handle large deformations. On the one hand, Lagrangian methods respect material interfaces and discontinuities but they are prone to severe mesh tangling and cannot capture large deformations, vorticity or shear. At best, these large mesh distortions result in a loss of accuracy but, in general, they cause an early breakdown of the calculations. On the other hand, Eulerian methodswhereby fluids flow over a fixed mesh — can handle such deformations but they fail to maintain sharp interfaces and produce diffusive solutions. ALE techniques combine the two previous approaches by controlling the mesh motion during the simulation. A full and recent review of ALE schemes is available in [8]. The advective terms introduced by this mesh motion can either (i) be taken into account directly in the PDEs (1) (ii) or be solved in a separate step [9]. The first case, usually termed "direct ALE" in the literature [10,11], is computationally less costly than the second one but introduces more complexity in the discretization step. Herein, we will only consider the second case, usually referred as "Lagrange-plus-remap", where the ALE algorithm is commonly split into three steps: (i) a Lagrangian update of variables whereby mesh cells are fluid elements carried and distorted by the flow; (ii) a rezoning (or regularization) phase in order to improve mesh cells' quality; and (iii) a remapping phase consisting in a conservative transfer (or advection) of the fields from the Lagrangian mesh to the rezoned mesh. More precisely, this paper deals with the step (iii) which is commonly a source of conservation and/or entropy errors. Indeed, a proper remapping stage in a multi-material hydrodynamic framework should respect some constraints [1, 2]:

- material locations: material interfaces should be preserved although they may not coincide with cell interfaces;

- conservation: mass, momentum and total energy have to be conserved;

- entropy control: remap has to be dissipative and entropy has to be conserved in smooth regions;

- monotonicity: remapped fields have to stay between justified bounds;

- continuity: fields have to remain unchanged when the mesh is not regularized;

- DeBar condition [4]: a constant velocity field has to be exactly remapped whatever the density field;

- accuracy: the remapping step has to be second-order in space on regular fields.

For space-staggered schemes, we have the additional need to remap node velocities in a compatible way. This question is addressed in [1,2] for flux-based staggered remap (see also [15] for an extension to axisymmetric geometry). The authors of [3] also proposed an intersection-based (or overlay-based) approach, instead of computing fluxes. The reader can refer to [16] for the multi-material extension of this work. Another direction consists in remapping on a finer grid where each cell is subdivided in nodal subcells [17]. 


\subsection{Conservation and entropy control in Lagrange-plus-remap algorithms}

Total energy conservation and entropy control play an important role for an accurate and robust computation of both shock-dominated and isentropic flows. Conservation of total energy is required in presence of strong shocks while entropy errors may lead to diffusion, overheating or lack of robustness and accuracy, especially in presence of non-linear equations of state. Keeping the energy conservation while controlling the entropy is challenging both for the Lagrangian phase and the remap. In more details, in a conservative context, a poor handling of the kinetic energy is the major source of entropy inconsistencies since the errors in kinetic and internal energies must compensate each other. More precisely, time and space discretization errors in the linear momentum equation introduce a numerical residual in the kinetic energy variation $\frac{d \mathcal{K}}{d t}$, i.e.

$$
\frac{d \mathcal{K}_{D}}{d t}+\int_{D} \sigma: \nabla u d V \neq 0,
$$

where $D$ is an isolated domain and $\sigma$ is the total stress tensor. For energy conservative Lagrangian schemes which strictly satisfy

$$
\frac{d}{d t}\left(\mathcal{K}_{D}+\int_{D} \rho e d V\right)=0,
$$

this induces an entropy violation in a reversible process, i.e.

$$
\int_{D} \rho T \frac{d s}{d t} d V:=\frac{d}{d t} \int_{D} \rho e d V-\int_{D} \sigma: \nabla \boldsymbol{u} d V=-\frac{d \mathcal{K}_{D}}{d t}-\int_{D} \sigma: \nabla \boldsymbol{u} d V \neq 0 .
$$

For entropy focused Lagrangian schemes which satisfy by construction

$$
\int_{D} \rho T \frac{d s}{d t} d V:=\frac{d}{d t} \int_{D} \rho e d V-\int_{D} \sigma: \nabla \boldsymbol{u} d V=0,
$$

errors in the kinetic energy variation lead to total energy conservation violation

$$
\frac{d}{d t}\left(\mathcal{K}_{D}+\int_{D} \rho e d V\right) \neq 0,
$$

which is inappropriate when handling strong shocks or large energy transfers. Kinetic energy errors can be removed in a conservative way by using consistent high-order non-linear implicit corrections in momentum equation [12,13] at the cost of an expensive algorithm. Note also the work of Burton et al. [14] on the reduction of dissipation in Cell-Centered Hydro $(\mathrm{CCH})$ schemes. But the issue is here to control such errors during the remap.

In practice, the kinetic energy is reconstructed on the new mesh with remapped velocities, corner and node masses. Two possibilities are then available to define the total energy on the new mesh: directly remap the total energy or remap the internal energy. On the one hand, if the total energy is projected, there might be a significative entropy error because the internal energy will not be controlled since it is deduced from the remapped total energy and the reconstructed kinetic energy. This error will be significative when dealing with large variations in kinetic and internal energies. On the other hand, if the internal energy is projected, the total energy will not be conserved since the reconstructed kinetic energy is not. As recommended in [17], we choose here internal energy as a remapped quantity in order to control entropy. A so-called "kinetic energy fix" or" De Bar fix" [4] is then required to retrieve the total energy conservation. But this fix is not associated to a pressure work and is therefore a source of entropy inducing an error since entropy-as any field—should be preserved during the remap. Consequently, to ensure a proper entropy control, this additional correction has to be positive- to ensure dissipation-and close to zero-at least to the scheme's order. To our knowledge, these two aspects are often overlooked, with the notable exception of [18] where a low-dissipation and flux-based algorithm (based on [19]) is developed thanks to considerations in momentum fluxes. A repair paradigm [20-22], possibly combined with monotonicity constraints [1], can also be used to overcome this drawback. An a posteriori re-distribution of fluxes is thus performed to enforce bounds preservation on several remapped quantities, including the internal energy. This procedure only considers the internal energy and does not fix the kinetic energy discrepancy. Moreover, the locality is lost if one wants to be independent of the order in which the re-distribution is performed, even if a few cells are concerned in principle [21]. It is finally worth mentioning that high-order staggered remap [23] — naturally motivated by high-order versions of Lagrangian SGH schemes-also represent a good way to decrease the kinetic energy error. 


\subsection{Overview of this work}

This paper deals with the analysis of the impact of a second-order staggered remap using an intersection-based (or overlay-based) approach on conservation properties and on the entropy control. We show that an accurate overlaybased remap with exact mesh intersections and exact integrations affects both the momentum and the kinetic energy because of node mass re-localizations and node velocity remap. We propose a staggered remapping strategy in order to take into account these discrepancies at a low computational cost. While preserving the strict conservation of total energy, our strategy allows to recover a proper entropy control at the expense of strict momentum conservation, "DeBar consistency" [4] and monotonicity losses. Concerning the entropy, the word "proper" is used here in the sense that our remap is dissipative - there is no destruction of entropy up to machine precision-and preserves isentropic flows to the scheme's order.

This staggered remapping strategy is then combined with a multi-material conservative space- and time-staggered (CSTS) Lagrangian scheme $[24,25]$ in order to numerically test the proposed strategy. This Lagrangian scheme strictly conserves mass, momentum, angular momentum and total energy [26, 27, 29]. It also guarantees an increase of entropy and its preservation in an isentropic process up to the scheme's order [24, 25, 30].

This paper is organized as follows. Our staggered remap is presented in Section 2, where exact intersections between meshes are performed. A correction step is proposed at that level in order to limit the entropic and energetic impact of this remap. The Lagrangian CSTS scheme [24, 25] — to be combined with our staggered remap-is described in Section 3 together with a summary of our full ALE algorithm. Numerical results are provided in Section 4 to assess the good entropic and energetic behavior of our full ALE approach.

\section{Staggered remap}

\subsection{Introduction and notation}

The goal of this section is to describe our multi-material staggered remap. In this framework, we will use a superscript

- "Old": for quantities defined on the original Lagrangian grid;

- "R": for quantities defined on the regularized grid directly after projection;

- "New": for quantities defined on the regularized grid after projection and correction as specified in §2.6.

In a given grid, cells (resp. nodes) are denoted by the index $c$ (resp. by $p$ ). Unless otherwise specified, sums over nodes or cells have to be understood as sums over all cells or all nodes on the entire grid, including boundaries. The set of all cells surrounding a node $p$ is denoted by $C(p)$ and all nodes of a cell $c$ are included in the set $P(c)$. The set of all nodes connected to a node $p$ by an edge or a cell is denoted by $Q(p)$.

\subsection{The remapping strategy}

From a theoretical point of view, an intersection-based remapping step, performed at an arbitrary time, reduces to the calculation of different volume integrals of various fields $\eta$ on a regularized grid. This grid is initially superposed with the Lagrangian computational grid. For any cell-centered scalar field $\eta$, the most general and robust strategy directly computes the new cell integrals

$$
\int_{V_{c}^{\mathrm{New}}} \eta d V=\sum_{c^{\prime}} \int_{V_{c}^{\mathrm{New}} \cap V_{c^{\prime}}^{\text {Old }}} \tilde{\eta}_{c^{\prime}}^{\text {Old }} d V,
$$

using a piecewise exact integration on each intersected piece $V_{c}^{\mathrm{New}} \cap V_{c^{\prime}}^{\text {Old }}$ between the new regularized mesh and the distorted Lagrangian grid. The notation $\tilde{\eta}_{c}^{\text {Old }}$ corresponds to the piecewise-linear approximation of the field $\eta$, on each Lagrangian cell, reconstructed from the mean values $\left\{\eta_{c}^{\text {Old }}\right\}$, i.e.

$$
\tilde{\eta}_{c}^{\text {Old }}(\boldsymbol{x})=\eta_{c}^{\text {Old }}+(\nabla \eta)_{c}^{\text {Old }} \cdot\left(\boldsymbol{x}-\left\langle\boldsymbol{x}>_{c}^{\text {Old }}\right),\right.
$$

where $\langle\boldsymbol{x}\rangle_{c}^{\text {Old }}$ is the geometric center of the Lagrangian cell $c$. This reconstruction allows to achieve a second-order accuracy [31]. The monotonicity on the quantity $\eta$ is ensured by the use of a non-linear mechanism, provided by 
Van Leer [32] in order to limit the gradient $(\nabla \eta)_{c}^{\text {Old }}$. This prohibits the apparition of new local extrema at the cost of a local loss of second-order accuracy.

The remap of any node field $\eta$ reuses the same method but on a dual mesh, where each node $p$ is associated to a cell $V_{p}$ obtained by joining the midpoints of the adjacent edges and the 2D cell centers of the adjacent cells (see Figure 1 for an example with quadrangular cells in 2D axisymmetric geometry). Node integrals after mesh regularization are then given by

$$
\int_{V_{p}^{\text {New }}} \eta d V=\sum_{p^{\prime}} \int_{V_{p}^{\text {New } \cap V_{p^{\prime}}^{\text {Old }}}} \tilde{\eta}_{p^{\prime}}^{\text {Old }} d V
$$

Again, the notation $\tilde{\eta}_{p}^{\text {Old }}$ corresponds to the piecewise-linear approximation of the field $\eta$, reconstructed on each dual cell from the mean values $\left\{\eta_{p}^{\text {Old }}\right\}$

$$
\tilde{\eta}_{p}^{\mathrm{Old}}(\boldsymbol{x})=\eta_{p}^{\mathrm{Old}}+(\boldsymbol{\nabla} \eta)_{p}^{\mathrm{Old}} \cdot\left(\boldsymbol{x}-<\boldsymbol{x}>_{p}^{\mathrm{Old}}\right),
$$

In the above formula, $\langle\boldsymbol{x}\rangle_{p}^{\text {Old }}$ is the geometric center of the dual Lagrangian cell $V_{p}^{\text {Old }}$ which is not necessarily the node Lagrangian position $\boldsymbol{x}_{p}^{\text {Old }}$. The constant term $\eta_{p}^{\text {Old }}$ in (9) should be the nodal cell average of $\eta$ and is thus $a$ priori not defined at $\boldsymbol{x}_{p}^{\text {Old }}$, possibly leading to a loss of accuracy if one replaces it by the nodal value of $\eta$. But a regular mesh assumption - as used in the proof of Relations (22) and (23), see Appendix B — ensures that $<\boldsymbol{x}>_{p}^{\text {Old }}$ and $\boldsymbol{x}_{p}^{\text {Old }}$ are within a distance of $O\left(\Delta x^{2}\right)$ and preserves the second order accuracy of (9). This will be confirmed by the numerical results of Section 4 on the isentropic vortex evolution. The regularity assumption will be however no longer valid in the cyclic remapping test presented at the end of the paper and a loss of accuracy will be observed.

From these integral constructions, we then define cell and node values on the regularized grid by

$$
\eta_{c}^{\text {New }}=\frac{1}{V_{c}^{\text {New }}} \int_{V_{c}^{\text {New }}} \tilde{\eta}^{\text {cell }} d V \text { and } \eta_{p}^{\text {New }}=\frac{1}{V_{p}^{\text {New }}} \int_{V_{p}^{\text {New }}} \tilde{\eta}^{\text {node }} d V
$$

where $\tilde{\eta}^{\text {cell }}$ (resp. $\tilde{\eta}^{\text {node }}$ ) stands for the cell (resp. nodal) reconstruction of Equation (7) (resp. of Equation (9)). Centered and staggered fields are conserved during the remap since by construction of $\left\langle\boldsymbol{x}>_{c}^{\text {Old }}\right.$ and $\langle\boldsymbol{x}\rangle_{p}^{\text {Old }}$ in $(7)$ and (9) the linear part of reconstructed fields are of zero average on the old cells

$$
\begin{aligned}
& \sum_{c} V_{c}^{\mathrm{New}} \eta_{c}^{\mathrm{New}}=\sum_{c} \int_{V_{c}^{\mathrm{New}}} \tilde{\eta} d V=\sum_{c} \int_{V_{c}^{\text {Old }}} \tilde{\eta} d V=\sum_{c} V_{c}^{\mathrm{Old}} \eta_{c}^{\mathrm{Old}}, \\
& \sum_{p} V_{p}^{\mathrm{New}} \eta_{p}^{\text {New }}=\sum_{p} \int_{V_{p}^{\text {New }}} \tilde{\eta} d V=\sum_{p} \int_{V_{p}^{\text {Old }}} \tilde{\eta} d V=\sum_{p} V_{p}^{\mathrm{Old}} \eta_{p}^{\text {Old }} .
\end{aligned}
$$

\subsection{Handling multimaterial cells}

The material dependent cell centered fields $\eta$ to be remapped are materials' density $\rho_{c}^{\varphi}$, volumic internal energy $(\rho e)_{c}^{\varphi}$ and Piola stress tensor $\tau_{c}^{\varphi}$. Remapping of vectors and tensors is done component by component by default since the symmetry preservation is not a major concern in the present paper. Centered fields are remapped for each material $\varphi$, which means that we must reduce our cell integration to the part $V_{c}^{\varphi}$,Old which is effectively occupied by the material $\varphi$. We therefore first need to introduce the volumic fraction $\alpha_{c}^{\varphi, \text { New }}$ of each material

$$
\alpha_{c}^{\varphi, \text { New }}=\frac{1}{V_{c}^{\mathrm{New}}} \sum_{c^{\prime}} \int_{V_{c}^{\mathrm{New}} \cap V_{c^{\prime}}^{\varphi, \text { Old }}} d V,
$$

before computing cell averaged values

$$
\eta_{c}^{\varphi, \text { New }}=\frac{1}{\alpha_{c}^{\varphi, \text { New }} V_{c}^{\mathrm{New}}} \sum_{c^{\prime}} \int_{V_{c}^{\text {New } \cap V_{c^{\prime}}^{\varphi, \text { Old }}}} \tilde{\eta}_{c^{\prime}}^{\varphi, \text { Old }} d V .
$$

A Volume-of-Fluid / Piecewise-Linear-Interface-Calculation (VoF/PLIC) interface reconstruction method [33] is used in order to construct the Lagrangian material subcells $V_{c}^{\varphi}$,Old . The reader can refer to [34] for a comparison 


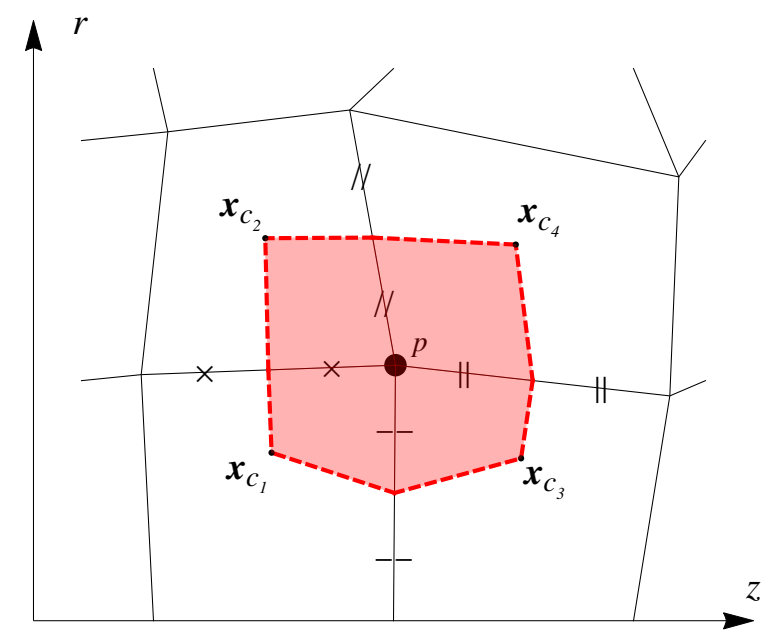

Figure 1: Dual cell $V_{p}$ built around a node $p$ for a quadrangular Lagrangian mesh in 2D axisymmetric geometry.

with some other interface reconstruction methods. An outgoing normal $\boldsymbol{n}_{c}^{\varphi, \text { Old }}$ is computed for each material $\varphi-$ defining the interface orientation—using volume fractions $\alpha_{c}^{\varphi, \text { Old }}$ in the multi-material Lagrangian cells [35]. Then, interfaces are located in the multi-material cell in order to match volume fractions $\alpha_{c}^{\varphi, \text { Old }}$ thanks to analytic formula available both in planar and axisymmetric geometries [36] which specifies the material subcell $V_{c}^{\varphi}$,Old . For simplicity, a first-order remap is performed in multi-material cells (as in boundary cells). The above material based reconstruction is still conservative since we have by construction

$$
\sum_{c} V_{c}^{\varphi, \text { New }} \eta_{c}^{\varphi, \text { New }}=\sum_{c} \alpha_{c}^{\varphi, \text { New }} V_{c}^{\text {New }} \eta_{c}^{\varphi, \text { New }}=\sum_{c} \int_{V_{c}^{\varphi, \text { old }}} \tilde{\eta}_{c}^{\varphi, \text { Old }} d V=\sum_{c} V_{c}^{\varphi, \text { Old }} \eta_{c}^{\varphi, \text { Old }} .
$$

A specific care must be given to internal energy reconstruction. Keeping in mind that the entropy field $s$ should be preserved during the remap - all the necessary entropy is deposed by the artificial viscosity in the Lagrangian phase-we construct herein an isentropic gradient of the internal energy [37-39] assuming that $\boldsymbol{\nabla} s=\mathbf{0}$. This leads to a specific formulation, derived in Appendix A, which is proportional to the limited density gradient

$$
(\boldsymbol{\nabla}(\rho e))_{c}^{\varphi, \text { Old }}=\left(e_{c}^{\varphi, \text { Old }}+\frac{p_{c}^{\varphi, \text { Old }}}{\rho_{c}^{\varphi, \text { Old }}}\right)(\nabla \rho)_{c}^{\varphi, \text { Old }} .
$$

Note that the above formula is valid for any equation of state. This choice relaxes the monotonicity constraint on the internal energy in order to enforce the preservation of isentropic flows up to second-order. The idea of introducing more physics in the limiting process at the expense of a strict monotonicity was already explored recently by Roe $e t$ al. in [40].

\subsection{Remap of node fields}

The node fields $\eta$ to be remapped are node density, momentum and kinetic energy, i.e. $\eta \in\left\{\rho_{p},(\rho \boldsymbol{u})_{p}, \mathcal{K}_{p}\right\}$. Let us recall that these staggered fields - including the node density $\rho_{p}$-are material-independant since we consider a unique velocity for all materials. The definition of node density $\rho_{p}$ is crucial for the accuracy and the continuity constraints outlined in Introduction. Driven by Lagrangian consistency, we choose here

$$
\rho_{p}^{\mathrm{Old}}=\frac{m_{p}^{\mathrm{Old}}}{V_{p}^{\mathrm{Old}}}, \quad(\rho \boldsymbol{u})_{p}^{\mathrm{Old}}=\rho_{p}^{\mathrm{Old}} \boldsymbol{u}_{p}^{\mathrm{Old}}, \quad \mathcal{K}_{p}^{\mathrm{Old}}=\frac{1}{2} \rho_{p}^{\mathrm{Old}}\left(\boldsymbol{u}_{p}^{\mathrm{Old}}\right)^{2}
$$

where $m_{p}$ is the node mass used in the Lagrangian step, mass which is usually built from the mass of the neighboring cells (see Subsection 3.1). Observe that the remap of this node density constructs a new node mass on the regularized 
mesh

$$
m_{p}^{\mathrm{R}}=\sum_{p^{\prime}} \int_{V_{p}^{\mathrm{New}} \cap V_{p^{\prime}}^{\text {old }}} \tilde{\rho}_{p^{\prime}}^{\mathrm{Old}} d V
$$

which differs from the cell based node mass $m_{p}^{\text {New }}$ to be used in the Lagrangian scheme, except when no rezoning is performed. In that case, all values $m_{p}^{\mathrm{R}}, m_{p}^{\mathrm{New}}$ and $m_{p}^{\text {Old }}$ are strictly equal thanks to the construction (16) of the node density $\rho_{p}$. This is akin with the approach of Kenamond et al. [15] developed in axisymmetric geometry.

The velocity after remap is finally obtained by

$$
\boldsymbol{u}_{p}^{\mathrm{R}}=\frac{V_{p}^{\mathrm{New}}}{m_{p}^{\mathrm{R}}}(\rho \boldsymbol{u})_{p}^{\mathrm{New}} .
$$

It must be stressed that here, the gradient $(\boldsymbol{\nabla}(\rho \boldsymbol{u}))_{p}^{\text {Old }}$ is based on an algebraic expansion

$$
(\boldsymbol{\nabla}(\rho \boldsymbol{u}))_{p}^{\mathrm{Old}}=\rho_{p}^{\mathrm{Old}}(\boldsymbol{\nabla} \boldsymbol{u})_{p}^{\mathrm{Old}}+(\boldsymbol{\nabla} \rho)_{p}^{\mathrm{Old}} \otimes \boldsymbol{u}_{p}^{\mathrm{Old}},
$$

in order to exactly conserve constant velocity fields even in presence of large or complex density gradients.

\subsection{Remapping errors}

The above remapping strategy can affect both the momentum and the kinetic energy in two ways. Let us denote $\mathcal{K}^{\mathrm{New}}=\sum_{p} \mathcal{K}_{p}^{\mathrm{New}}$ the global kinetic energy after remap which exactly matches the global kinetic energy before remap due to (11).

Error due to the linear momentum remap. Both the reconstruction and the integral averaging modify the mass weighted $L^{2}$-norm of the velocity, i.e. $\mathcal{K}^{\mathrm{New}} \neq \sum_{p} \frac{1}{2} m_{p}^{\mathrm{R}}\left(\boldsymbol{u}_{p}^{\mathrm{R}}\right)^{2}$, with a kinetic energy increase during the reconstruction step and a kinetic energy decrease during the remap. The difference $\mathcal{K}^{\text {New }}-\sum_{p} \frac{1}{2} m_{p}^{\mathrm{R}}\left(\boldsymbol{u}_{p}^{\mathrm{R}}\right)^{2}$ is not positive a priori which can be a problem for entropy control.

Error due to the mass transfer. As previously explained, the node mass $m_{p}^{\mathrm{New}}$, used in the Lagrangian phase and reconstructed on the new mesh, is built from the mass of the neighboring cells $m_{c}^{\mathrm{New}}$ and differs from the node mass $m_{p}^{\mathrm{R}}$ obtained on the dual mesh by remapping. There is therefore a mass re-localization after remap which induces a change in the linear momentum and an additional transfer of kinetic energy

$$
\begin{gathered}
\sum_{p} m_{p}^{\mathrm{R}} \boldsymbol{u}_{p}^{\mathrm{R}} \neq \sum_{p} m_{p}^{\mathrm{New}} \boldsymbol{u}_{p}^{\mathrm{R}}, \\
\mathcal{K}^{\mathrm{New}} \neq \sum_{p} \frac{1}{2} m_{p}^{\mathrm{R}}\left(\boldsymbol{u}_{p}^{\mathrm{R}}\right)^{2} \neq \sum_{p} \frac{1}{2} m_{p}^{\mathrm{New}}\left(\boldsymbol{u}_{p}^{\mathrm{R}}\right)^{2} .
\end{gathered}
$$

Again, the sign of the expression $\sum_{p} \frac{1}{2} m_{p}^{\mathrm{R}}\left(\boldsymbol{u}_{p}^{\mathrm{R}}\right)^{2}-\sum_{p} \frac{1}{2} m_{p}^{\text {New }}\left(\boldsymbol{u}_{p}^{\mathrm{R}}\right)^{2}$ is not controlled a priori.

On smooth meshes, both modifications can be proved to be second-order in space, i.e.

$$
\begin{aligned}
& m_{p}^{\text {New }}-m_{p}^{\mathrm{R}}=m_{p}^{\mathrm{R}} \times O\left(\Delta \boldsymbol{x}^{2}\right), \\
& \mathcal{K}_{p}^{\text {New }}-\frac{1}{2} m_{p}^{\mathrm{R}}\left(\boldsymbol{u}_{p}^{\mathrm{R}}\right)^{2}=\mathcal{K}_{p}^{\text {New }} \times O\left(\Delta \boldsymbol{x}^{2}\right),
\end{aligned}
$$

with the exception of the axisymmetric geometry where the second-order term $O\left(\Delta \boldsymbol{x}^{2}\right)$ becomes $O(\Delta x) \times O\left(\frac{\Delta r}{r}\right)$, inducing therefore a loss of accuracy next to the axis $r=0$. Appendix B checks Relations (22) and (23) for the particular choice of node mass $m_{p}$ used in our test cases.

Note finally that, in the absence of rezoning, the node density definition (16) guarantees that linear momentum and kinetic energy will be strictly preserved (continuity constraint). 


\subsection{Correction phase}

Inequalities (20) and (21) show that the above remap violates momentum and total energy conservations. The goal of the correction phase is to restore energy conservation in a local entropy controlled way and at a low computational cost. The basic idea is to introduce the local kinetic energy variation into the local internal energy as proposed by DeBar [4]. Before this, it is useful to perform a velocity correction in order to minimize this kinetic energy variation, and hence the resulting entropy error. We propose herein to correct the velocity by

$$
\boldsymbol{u}_{p}^{\mathrm{New}}=\lambda_{p} \boldsymbol{u}_{p}^{\mathrm{R}}, \text { where } \lambda_{p}=\sqrt{\frac{\min \left(\mathcal{K}_{p}^{\mathrm{New}}, \frac{1}{2} m_{p}^{\mathrm{R}}\left(\boldsymbol{u}_{p}^{\mathrm{R}}\right)^{2}\right)}{\frac{1}{2} m_{p}^{\mathrm{New}}\left(\boldsymbol{u}_{p}^{\mathrm{R}}\right)^{2}}} .
$$

This correction cancels the kinetic energy variation when strict energy conservation cannot be retrieved in an entropic way. Observe that $\lambda_{p}=1$ in the absence of remapping where we have $m_{p}^{\text {Old }}=m_{p}^{\mathrm{R}}=m_{p}^{\text {New }}, \boldsymbol{u}_{p}^{\text {Old }}=\boldsymbol{u}_{p}^{\mathrm{R}}$ and $\mathcal{K}_{p}^{\text {New }}=$ $\mathcal{K}_{p}^{\mathrm{Old}}=\frac{1}{2} m_{p}^{\mathrm{R}}\left(\boldsymbol{u}_{p}^{\mathrm{R}}\right)^{2}$, which ensures the continuity of the correction (24) with respect to mesh motion. It is explicit and second-order in space in smooth regions (except near the axis $r=0$ in axisymmetric geometry) according to Relations (22) and (23). With this new definition of velocity, the internal energy is then corrected by

$$
\begin{aligned}
& m_{c}^{\varphi, \text { New }} e_{c}^{\varphi, \text { New }}=m_{c}^{\varphi, \text { New }} e_{c}^{\varphi, \mathrm{R}}+\delta \mathcal{K}_{c}^{\varphi, \text { New }}, \\
& \delta \mathcal{K}_{c}^{\varphi, \text { New }}=\sum_{p \in P(c)} \frac{m_{c p}^{\varphi, \text { New }}}{m_{p}^{\text {New }}}\left(\mathcal{K}_{p}^{\text {New }}-\frac{1}{2} m_{p}^{\text {New }}\left(\boldsymbol{u}_{p}^{\text {New }}\right)^{2}\right) .
\end{aligned}
$$

The node kinetic energy discrepancy is distributed here among cells $c \in C(p)$ and materials $\varphi$ thanks to a user defined mass ratio $\frac{m_{c}^{\varphi} \text { New }}{m_{p}^{\text {New }}}$ measuring after projection the relative mass contribution of the material $\varphi$ in cell $c$ to the node $p$. This distribution process is not unique with different examples given in [2]. In our applications, we use the cell material mass $m_{c}^{\varphi}$ and the corner volumes $V_{c p}^{\text {mass }}$ defined in (30) and set

$$
m_{c p}^{\varphi}=\frac{V_{c p}^{\text {mass }}}{V_{c}} m_{c}^{\varphi}, \quad m_{p}=\sum_{c \in C(p)} \sum_{\varphi} m_{c p}^{\varphi} .
$$

Corrections (24), (25) and (26) extends the philosophy of the CSTS Lagrangian scheme [24], presented in Section 3 , where a consistent correction is introduced in the linear momentum equation in order to preserve the local kinetic energy. Let us now prove the consistency and the proper entropy control of the above corrections.

Consistency. If $\mathcal{K}_{p}^{\mathrm{New}}>\frac{1}{2} m_{p}^{\mathrm{R}}\left(\boldsymbol{u}_{p}^{\mathrm{R}}\right)^{2}$, we have

$$
\lambda_{p}=\sqrt{\frac{\frac{1}{2} m_{p}^{\mathrm{R}}\left(\boldsymbol{u}_{p}^{\mathrm{R}}\right)^{2}}{\frac{1}{2} m_{p}^{\text {New }}\left(\boldsymbol{u}_{p}^{\mathrm{R}}\right)^{2}}}=\sqrt{\frac{m_{p}^{\mathrm{R}}}{m_{p}^{\text {New }}}}=\sqrt{1+O\left(\Delta x^{2}\right)}=1+O\left(\Delta x^{2}\right),
$$

thanks to Relation (22). Otherwise, when $\mathcal{K}_{p}^{\mathrm{New}}<\frac{1}{2} m_{p}^{\mathrm{R}}\left(\boldsymbol{u}_{p}^{\mathrm{R}}\right)^{2}$, we get from (23) and (22)

$$
\lambda_{p}=\sqrt{\frac{\mathcal{K}_{p}^{\text {New }}}{\frac{1}{2} m_{p}^{\text {New }}\left(\boldsymbol{u}_{p}^{\mathrm{R}}\right)^{2}}}=\sqrt{\frac{\frac{1}{2} m_{p}^{\mathrm{R}}\left(\boldsymbol{u}_{p}^{\mathrm{R}}\right)^{2}\left(1+O\left(\Delta x^{2}\right)\right)}{\frac{1}{2} m_{p}^{\text {New }}\left(\boldsymbol{u}_{p}^{\mathrm{R}}\right)^{2}}}=1+O\left(\Delta x^{2}\right) .
$$

In both cases, we therefore have

$$
\lambda_{p}=1+O\left(\Delta x^{2}\right)
$$

Anticipating the discussion in Subsection 3.3, one can already notice that the above Equation (27) has a strong impact on the "DeBar condition" and the momentum conservation. The velocity correction breaks the strict momentum conservation which is now conserved to the second-order. Concerning the "DeBar condition", even in the case of a constant velocity $\boldsymbol{u}_{p}^{\text {Old }}=\boldsymbol{u} \forall p, \lambda_{p}=\sqrt{\frac{m_{p}^{\mathrm{R}}}{m_{p}^{\text {New }}}}$ might be different than one due to density gradient. 
Proper entropy control. Equation (24) allows to write

$$
\frac{1}{2} m_{p}^{\text {New }}\left(\boldsymbol{u}_{p}^{\text {New }}\right)^{2}=\frac{1}{2} m_{p}^{\text {New }} \lambda_{p}^{2}\left(\boldsymbol{u}_{p}^{\mathrm{R}}\right)^{2}=\min \left(\mathcal{K}_{p}^{\text {New }}, \frac{1}{2} m_{p}^{\mathrm{R}}\left(\boldsymbol{u}_{p}^{\mathrm{R}}\right)^{2}\right)
$$

Thanks to Relations (26)-(27)-(28), it is then straightforward to show that the above velocity correction results in a positive and second-order kinetic energy discrepancy

$$
\delta \mathcal{K}_{c}^{\varphi, \text { New }} \geq 0 \text { and } \delta \mathcal{K}_{c}^{\varphi, \text { New }}=m_{c}^{\varphi, \text { New }} \times O\left(\Delta x^{2}\right),
$$

and hence a proper entropy control. Moreover, since $\mathcal{K}_{p}^{\mathrm{New}}$ and $\frac{1}{2} m_{p}^{\mathrm{R}}\left(\boldsymbol{u}_{p}^{\mathrm{R}}\right)^{2}$ use the same reconstruction procedure, we expect this kinetic energy discrepancy to be very small. In the above relations, second-order residues $O\left(\Delta x^{2}\right)$ have to be replaced in axisymmetric geometry by $O(\Delta x) \times O\left(\frac{\Delta r}{r}\right)$, inducing a loss of accuracy next to the axis $r=0$.

\section{Our ALE algorithm}

\subsection{CSTS Lagrangian step}

We now want to couple this general remapping strategy with a specific Lagrangian solver. We focus here on numerical schemes which are space and time staggered [24, 29, 62] where thermodynamic quantities (material density, material internal energy, material pressure) are defined at cell centers and at time $t^{n}$ while kinematic quantites (velocities) are defined at nodes and calculated at half time $t^{n+1 / 2}$. In what follows, the superscript $n+1 / 2$ refers to time-staggered quantities while $n$ or $n+1$ refer to time-centered ones.

Such staggered schemes have nice entropic properties and can preserve total energy [28, 29]. But, when combining our remapping strategy with these time staggered algorithms, there is an issue on the time at which the remap has to be performed. Taking into account the key role played by the kinetic energy in energy conservation and entropy control, we decide to perform the remap at half time $t^{n+1 / 2}$ where velocities, node masses - and therefore the kinetic energyare naturally defined, and where the internal energy can be easily reconstructed. An overview of the whole ALE algorithm is given in the next Subsection 3.2 highlighting this uncommon choice.

To illustrate this point, we briefly review the CSTS Lagrangian hydrodynamic scheme [24, 25] which we will use in combination with the above remapping strategy. First, we need to define the node masses to be used in the linear momentum equation. Below, node masses are computed from the cell masses $m_{c}$ according to the geometric partition rule

$$
m_{p}=\sum_{c \in C(p)} m_{c p}, \quad \text { where } \quad m_{c p}=\frac{V_{c p}^{\text {mass }}}{V_{c}} m_{c} \quad \text { and } \quad V_{c p}^{\text {mass }}=\frac{2}{3} V_{c p}^{\mathrm{M}}+\frac{1}{3} V_{c p}^{\mathrm{W}} .
$$

The corner volume $V_{c p}^{\mathrm{M}}$ is delimited by the "so-called" median mesh $[43,44]$ and $V_{c p}^{\mathrm{W}}=\frac{1}{|P(c)|} V_{c}$ is the volume defined by Kolsky and Wilkins $[5,45]$. This corner volume $V_{c p}^{\text {mass }}$ is different from the volume $V_{c p}=V_{p} \cap V_{c}$ generated by intersecting the dual node cell $V_{p}$ used in Section 2 for remap with the primal cell $V_{c}$. Note that this corresponds to a "volume-weighted" momentum formulation since (30) in axisymmetric geometry uses the volumes of the torus generated by rotation of individual cells or subcells around the $z$ axis. Definition (30) strictly preserves the planar symmetry on a cartesian mesh and at a given time in axisymmetric geometry [24, 25, 27]. Consider a pure axial 1D Sod shock tube [48] in 2D axisymmetric geometry-described in Figure 2(a)—where the three classical waves propagate along the axial direction without any radial motion. Only the linear combination in Equation (30) gives a $r$-independant and purely axial acceleration on a uniform mesh [27] as shown in Figure 2. Details are given in [24] and the demonstration is avalaible in [25, Chapter 3, Section 3.7]. The space and time discretization of the linear momentum conservation law is then obtained from the least action principle applied on a discrete action integral [29]. Since the discrete momentum equation is implicit with respect to the velocity $\boldsymbol{u}_{p}^{n+1 / 2}$ and the node mass $m_{p}^{n+1 / 2}$, it is solved by a four steps predictor corrector algorithm [24, 25]:

1. Node mass prediction $m_{p}^{* n+1 / 2}$

$$
m_{p}^{* n+1 / 2}=m_{p}^{n-1 / 2}+\left.\sum_{q \in Q(p)} \frac{\partial m_{p}}{\partial x_{q}}\right|^{n-1 / 2} \cdot \boldsymbol{u}_{q}^{n-1 / 2} \frac{\Delta t^{n+1 / 2}+\Delta t^{n-1 / 2}}{2} .
$$

The calculation details of corner mass vectors $\frac{\partial m_{p}}{\partial x_{q}}$ can be found in [25]. 
2. Velocity prediction $\boldsymbol{u}_{p}^{* n+1 / 2}$ using second order kinetic energy control

$$
\begin{aligned}
& m_{p}^{* n+1 / 2} \boldsymbol{u}_{p}^{* n+1 / 2}-m_{p}^{n-1 / 2} \boldsymbol{u}_{p}^{n-1 / 2}=\sum_{c \in C(p)}\left[\left.\left(p_{c}^{n}+q_{c}^{n-1 / 2}\right) \frac{\partial V_{c}}{\partial x_{p}}\right|^{n}+\boldsymbol{f}_{c p}^{n-1 / 2}\right] \frac{\Delta t^{n+1 / 2}+\Delta t^{n-1 / 2}}{2} \\
& +\left.\sum_{q \in Q(p)} \frac{1}{4}\left(\boldsymbol{u}_{q}^{n-1 / 2}\right)^{2} \frac{\partial m_{q}}{\partial x_{p}}\right|^{n-1 / 2} \Delta t^{n-1 / 2}+\left.\sum_{q \in Q(p)} \frac{1}{4}\left(\boldsymbol{u}_{q}^{n-1 / 2}\right)^{2} \frac{\partial m_{q}}{\partial x_{p}}\right|^{n-1 / 2} \Delta t^{n+1 / 2},
\end{aligned}
$$

where $q_{c}^{n-1 / 2}=\underline{Q}\left[\left\{\boldsymbol{u}_{p}^{n-1 / 2}\right\}\right]$ is the artificial viscosity and $\boldsymbol{f}_{c p}^{n-1 / 2}=\mathcal{F}\left[\left\{\boldsymbol{u}_{p}^{n-1 / 2}\right\}\right]$ corresponds to the anti-hourglassing force.

3. Geometric update

4. Velocity correction

$$
\begin{aligned}
& \boldsymbol{x}_{p}^{* n+1 / 2}=\boldsymbol{x}_{p}^{n}+\boldsymbol{u}_{p}^{* n+1 / 2} \frac{\Delta t^{n+1 / 2}}{2}, \\
& m_{p}^{n+1 / 2}=m\left[\left\{\boldsymbol{x}_{p}^{* n+1 / 2}\right\}\right] \quad \text { and calculation of }\left.\quad \frac{\partial m_{q}}{\partial \boldsymbol{x}_{p}}\right|^{n+1 / 2}
\end{aligned}
$$

$$
\begin{aligned}
m_{p}^{n+1 / 2} \boldsymbol{u}_{p}^{n+1 / 2}-m_{p}^{n-1 / 2} \boldsymbol{u}_{p}^{n-1 / 2}=\sum_{c \in C(p)}[ & \left.\left.\left(p_{c}^{n}+q_{c}^{n}\right) \frac{\partial V_{c}}{\partial x_{p}}\right|^{n}+\boldsymbol{f}_{c p}^{n}\right] \frac{\Delta t^{n+1 / 2}+\Delta t^{n-1 / 2}}{2} \\
& +\left.\sum_{q \in Q(p)} \frac{1}{4}\left(\boldsymbol{u}_{q}^{n-1 / 2}\right)^{2} \frac{\partial m_{q}}{\partial x_{p}}\right|^{n-1 / 2} \Delta t^{n-1 / 2}+\left.\sum_{q \in Q(p)} \frac{1}{4}\left(\boldsymbol{u}_{q}^{* n+1 / 2}\right)^{2} \frac{\partial m_{q}}{\partial x_{p}}\right|^{n+1 / 2} \Delta t^{n+1 / 2},
\end{aligned}
$$

where $q_{c}^{n}=\underline{Q}\left[\left\{\frac{1}{2}\left(\boldsymbol{u}_{p}^{* n+1 / 2}+\boldsymbol{u}_{p}^{n-1 / 2}\right)\right\}\right]$ and $\boldsymbol{f}_{c p}^{n}=\mathcal{F}\left[\left\{\frac{1}{2}\left(\boldsymbol{u}_{p}^{* n+1 / 2}+\boldsymbol{u}_{p}^{n-1 / 2}\right)\right\}\right]$.

Above, pressure forces have been complemented by an artificial viscosity term $q_{c}^{\varphi}$ and variational inertial based antihourglassing forces $f_{c p}^{\varphi}$ in order to handle strong shocks and reduced integration. These dissipative terms are beyond the scope of this paper and are given in Appendix C. The simple equi-compressibility closure model $[41,42]$ gives the relation $p_{c}=\sum_{\varphi} \alpha_{c}^{\varphi} p_{c}^{\varphi}$ in multi-material cells where $\alpha_{c}^{\varphi}$ is the constant volume fraction for material $\varphi$. Material volume variations $\left.\frac{\partial V_{c}}{\partial x_{p}}\right|^{\varphi}$ are thus determined by $\left.\frac{\partial V_{c}}{\partial x_{p}}\right|^{\varphi}=\alpha_{c}^{\varphi} \frac{\partial V_{c}}{\partial x_{p}}$.

The internal energy evolution from times $t^{n}$ to $t^{n+1}$ is then deduced from an energy conservation principle written at time $t^{n+1 / 2}$, using the "flux-in-time" approach of [29]. In order to be able to remap the internal energy $e_{c}^{\varphi, n+1 / 2}$ at half time steps, the material internal energy is updated into two phases. The first one is explicit and constructs the internal energy $e_{c}^{\varphi, n+1 / 2}$ at half time step

$$
\begin{aligned}
m_{c}^{\varphi}\left(e_{c}^{\varphi, n+1 / 2}-e_{c}^{\varphi, n}\right)=\sum_{p \in P(c)}\{ & -\frac{1}{2}\left[\left.\left(p_{c}^{\varphi, n}+q_{c}^{\varphi, n}\right) \frac{\partial V_{c}}{\partial x_{p}}\right|^{\varphi, n}+\boldsymbol{f}_{c p}^{\varphi, n}\right] \cdot \boldsymbol{u}_{p}^{n+1 / 2} \Delta t^{n+1 / 2} \\
+ & \frac{1}{4}\left[\left.\left(p_{c}^{\varphi, n}+q_{c}^{\varphi, n}\right) \frac{\partial V_{c}}{\partial x_{p}}\right|^{\varphi, n}+\boldsymbol{f}_{c p}^{\varphi, n}\right] \cdot\left(\boldsymbol{u}_{p}^{n+1 / 2}-\boldsymbol{u}_{p}^{n-1 / 2}\right)\left(\Delta t^{n+1 / 2}-\Delta t^{n-1 / 2}\right) \\
& -\frac{1}{2}\left[\left.\left(q_{c}^{\varphi, n}-q_{c}^{\varphi, n-1 / 2}\right) \frac{\partial V_{c}}{\partial x_{p}}\right|^{\varphi, n}+\boldsymbol{f}_{c p}^{\varphi, n}-\boldsymbol{f}_{c p}^{\varphi, n-1 / 2}\right] \cdot \boldsymbol{u}_{p}^{n-1 / 2} \Delta t^{n-1 / 2} \\
& \quad-\left.\sum_{q \in P(c)} \frac{1}{8}\left(\boldsymbol{u}_{q}^{n-1 / 2}\right)^{2} \frac{\partial m_{c q}}{\partial x_{p}}\right|^{\varphi, n-1 / 2} \cdot\left(\boldsymbol{u}_{p}^{n+1 / 2}+\boldsymbol{u}_{p}^{n-1 / 2}\right) \Delta t^{n-1 / 2} \\
& -\left.\sum_{q \in P(c)} \frac{1}{8}\left(\boldsymbol{u}_{q}^{* n+1 / 2}\right)^{2} \frac{\partial m_{c q}}{\partial x_{p}}\right|^{\varphi, n+1 / 2} \cdot\left(\boldsymbol{u}_{p}^{n+1 / 2}+\boldsymbol{u}_{p}^{n-1 / 2}\right) \Delta t^{n+1 / 2} \\
& \left.+\frac{1}{2}\left(m_{c p}^{\varphi, n+1 / 2}-m_{c p}^{\varphi, n-1 / 2}\right) \boldsymbol{u}_{p}^{n+1 / 2} \cdot \boldsymbol{u}_{p}^{n-1 / 2}\right\} .
\end{aligned}
$$

The second one is locally implicit, and updates the internal energy on each cell by solving a $2 \times 2$ non-linear system with unknowns $e_{c}^{\varphi, n+1}$ and $p_{c}^{\varphi, n+1}$

$$
m_{c}^{\varphi}\left(e_{c}^{\varphi, n+1}-e_{c}^{\varphi, n+1 / 2}\right)=\sum_{p \in P(c)}-\frac{1}{2}\left[\left.\left(p_{c}^{\varphi, n+1}+q_{c}^{\varphi, n+1 / 2}\right) \frac{\partial V_{c}}{\partial x_{p}}\right|^{\varphi, n+1}+\boldsymbol{f}_{c p}^{\varphi, n+1 / 2}\right] \cdot \boldsymbol{u}_{p}^{n+1 / 2} \Delta t^{n+1 / 2},
$$




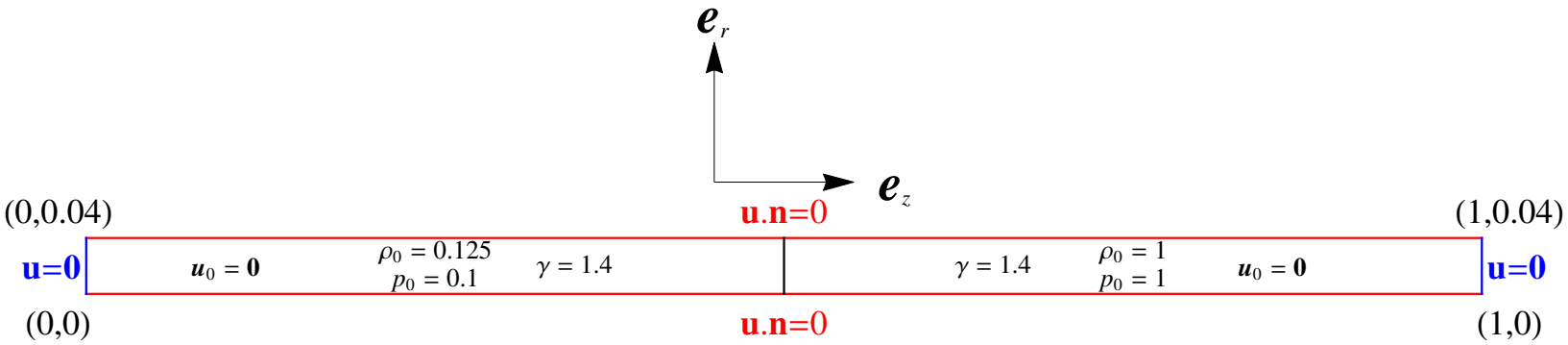

(a)

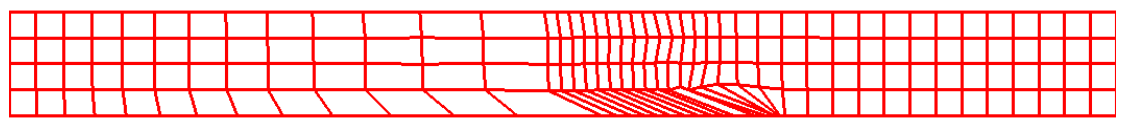

(b)

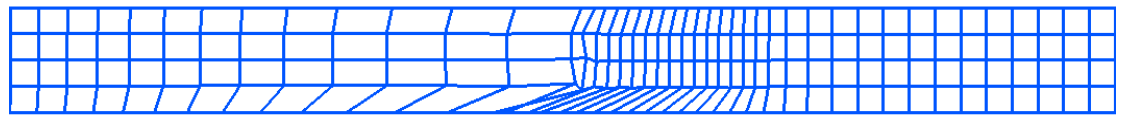

(c)

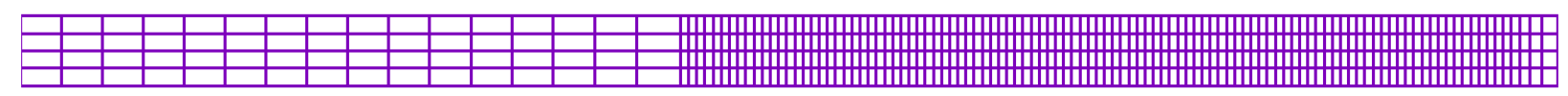

(d)

Figure 2: Pure axial (1D) Sod shock tube [48] in axisymmetric geometry: (a) initial and boundary conditions; (b) last mesh computed with the corner volume $V_{c p}^{\mathrm{M}}[43,44]$ before premature stop of the calculation; (c) last mesh computed with the corner volume $V_{c p}^{\mathrm{W}}[5,45]$ before premature stop of the calculation; and (d) final mesh at $t=2$ computed with the corner volume $V_{c p}^{\text {mass }}$ of Definition (30). Strong singularities near the axis $r=0$ appear for cases (b) and (c) leading to calculation failure before reaching the final time while the axial 1D symmetry is strictly preserved in case (d).

$$
p_{c}^{\varphi, n+1}=\operatorname{EOS}_{\varphi}\left(\rho_{c}^{\varphi, n+1}, e_{c}^{\varphi, n+1}\right) .
$$

In the above formula, the node anti-hourglassing forces have been split into local contribution $\boldsymbol{f}_{c p}^{\varphi}$ based on the mass weighting factor $\frac{m_{c}^{\varphi}}{m_{c}}$.

In Equations (32), (35), (36) and (37), subscripts $p$ and $q$ are node indexes—-see Subsection 2.1—and do not refer to pressure and artificial viscosity also denoted by $p$ and $q$.

\subsection{Lagrange-plus-remap CSTS scheme}

By combining the remap of Section 2 with the above lagrangian scheme, we get the following algorithm

\section{Lagrangian step [24]}

1. Time step calculation $\Delta t^{n+1 / 2}$ between times $t^{n}$ and $t^{n+1}$

$$
\Delta t^{n+1 / 2}=\mathrm{CFL} \times \min _{c}\left\{\ell_{c}^{n} / c_{c}^{n}\right\}
$$

where $\ell_{c}^{n}$ is a characteristic length, $c_{c}^{n}$ is the sound speed in a cell $c$ and CFL is a user-defined coefficient

2. Velocity prediction $\boldsymbol{u}_{p}^{*, n+1 / 2}$

3. Geometry prediction $\boldsymbol{x}_{p}^{*, n+1 / 2}$

4. Calculation of the corresponding dissipative terms $\mathcal{D}_{c}^{\varphi, n}=\left\{q_{c}^{\varphi, n},\left\{\boldsymbol{f}_{c p}^{\varphi, n}\right\}_{p \in P(c)}\right\}$

5. Calculation of the node mass and its variations

$$
\mathcal{M}_{p}^{n+1 / 2, \text { Old }}=\left\{\left\{m_{c p}^{n+1 / 2, \text { Old }}\right\}_{c \in C(p)},\left\{\left.\frac{\partial m_{c q}}{\partial \boldsymbol{x}_{p}}\right|^{n+1 / 2, \text { Old }}\right\}_{c \in C(p), q \in P(c)}\right\}
$$


6. Velocity update $\boldsymbol{u}_{p}^{n+1 / 2}$

7. Node position update

$$
\boldsymbol{x}_{p}^{n+1 / 2}=\boldsymbol{x}_{p}^{n}+\boldsymbol{u}_{p}^{n+1 / 2} \frac{\Delta t^{n+1 / 2}}{2}
$$

8. Internal energy calculation $e_{c}^{n+1 / 2, \varphi}$

Rezoning step at time $t^{n+1 / 2}$

9. Grid smoothing $\boldsymbol{x}_{p}^{n+1 / 2}$ in case of excessive mesh distorsion

Remapping step at time $t^{n+1 / 2}$

10. Remap of cell-centered variables: volumic fractions $\alpha_{c}^{\varphi}$, material mass $\rho_{c}^{\varphi, n+1 / 2}$ and material internal energy $(\rho e)_{c}^{\varphi, n+1 / 2, \mathrm{R}}$

11. Calculation of the node mass and its variations on the new geometry

$$
\mathcal{M}_{p}^{n+1 / 2, \mathrm{New}}=\left\{\left\{m_{c p}^{n+1 / 2, \mathrm{New}}\right\}_{c \in C(p)},\left\{\left.\frac{\partial m_{c q}}{\partial \boldsymbol{x}_{p}}\right|^{n+1 / 2, \mathrm{New}}\right\}_{c \in C(p), q \in P(c)}\right\}
$$

12. Remap of node-centered values: node mass $\rho_{p}^{n+1 / 2}$, node momentum $(\rho \boldsymbol{u})_{p}^{n+1 / 2, \mathrm{R}}$ and node kinetic energy $\mathcal{K}_{p}^{n+1 / 2}$

Remap correction step at $t^{n+1 / 2}$

13. Calculation of the post-remap node mass $m_{p}^{n+1 / 2, \mathrm{R}}$

14. Velocity correction $\boldsymbol{u}_{p}^{n+1 / 2, \text { New }}$ to handle node mass re-localization

15. Internal energy correction $e_{c}^{\varphi, n+1 / 2, \mathrm{New}}$ to cancel energy remapping errors.

Lagrangian step [24]

16. Node position update

$$
\boldsymbol{x}_{p}^{n+1}=\boldsymbol{x}_{p}^{n+1 / 2}+\boldsymbol{u}_{p}^{n+1 / 2, \mathrm{New}} \frac{\Delta t^{n+1 / 2}}{2}
$$

17. Calculation of the associated dissipative terms

$$
\mathcal{D}_{c}^{\varphi, n+1 / 2}=\left\{q_{c}^{\varphi, n+1 / 2},\left\{\boldsymbol{f}_{c p}^{\varphi, n+1 / 2}\right\}_{p \in P(c)}\right\}
$$

18. Final update of the material internal energies $e_{c}^{\varphi, n+1}$, together with the material pressures $p_{c}^{\varphi, n+1}$

19. Average internal energy and pressure calculation $e_{c}^{n+1}$ and $p_{c}^{n+1}$ within each multi-material cell using the equicompressibility closure model

Note that the node mass $m_{p}^{n+1 / 2}$ and its variations are computed on the predicted geometry $\boldsymbol{x}_{p}^{*, n+1 / 2}$ and not on the geometry $\boldsymbol{x}_{p}^{n+1 / 2}$ defined by (40). Indeed, the difference between the two geometries is a second-order residue since $\boldsymbol{x}_{p}^{*, n+1 / 2}=\boldsymbol{x}_{p}^{n+1 / 2}+O\left(\Delta t^{3}\right)$. This prevents to compute these geometrical terms twice during the Lagrangian phase. The above algorithm is also summarized in Tab. 1 where each step is associated to its corresponding equation number. 


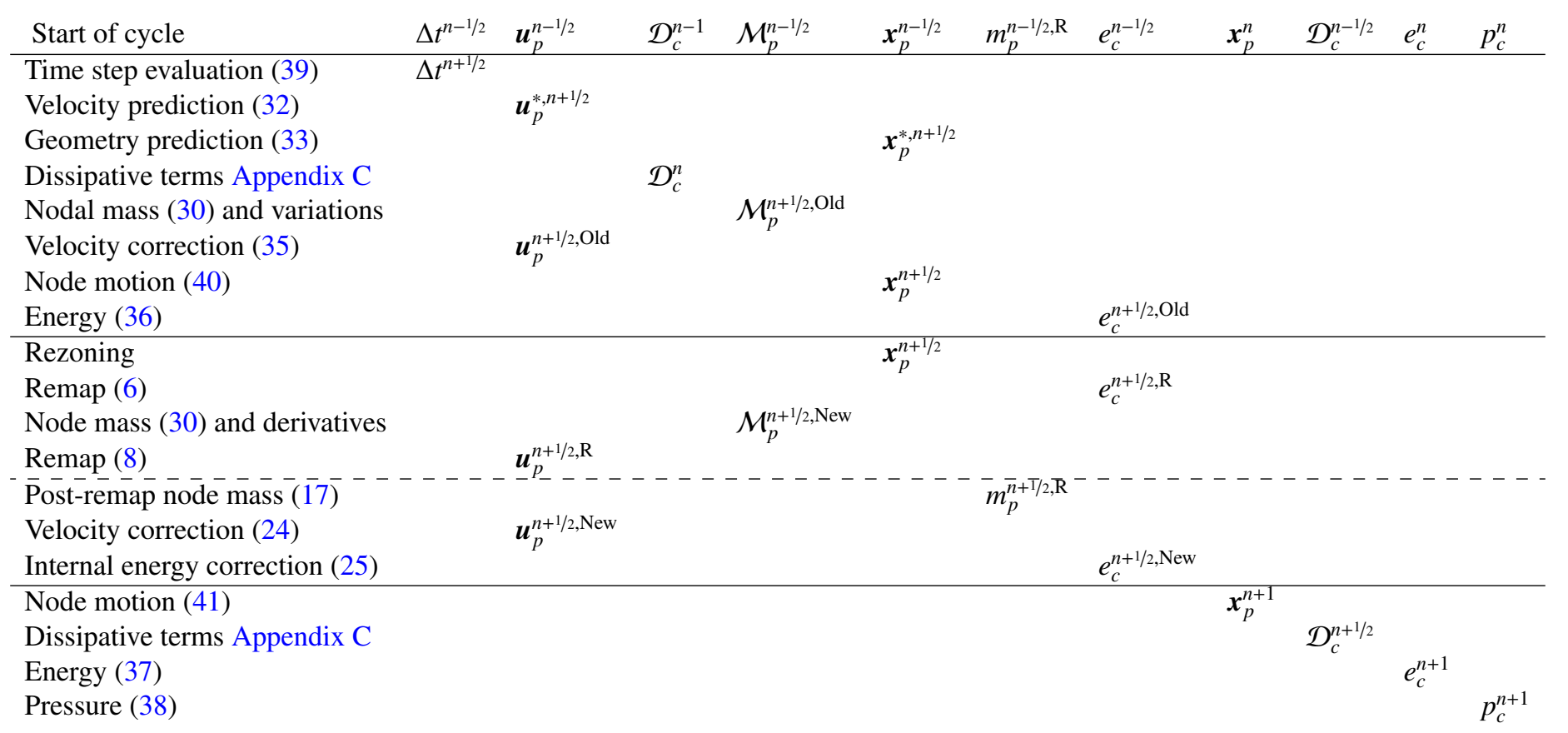

Table 1: Global ALE algorithm: the principal variables in the scheme are indicated in the order of evaluation in the time loop with the corresponding equation number. Dissipative terms, corner masses and their derivatives are gathered in variables $\mathcal{D}_{c}=\left\{q_{c},\left\{\boldsymbol{f}_{c p}\right\}_{p \in P(c)}\right\}$ and $\mathcal{M}_{p}=\left\{\left\{m_{c p}\right\}_{c \in C(p)},\left\{\frac{\partial m_{c q}}{\partial x_{p}}\right\}_{c \in C(p), q \in P(c)}\right\}$. Only global values are indicated for multi-material cells. Solid lines separate instantaneous rezoning, remapping and correction phases from the Lagrangian update, corresponding to a time evolution of the system. Dashed lines separate the rezoning and remapping phases from the correction phase. 


\subsection{Properties of the ALE algorithm}

By construction of both Lagrangian and remapping steps, the whole ALE scheme is second-order accurate in space and time on smooth grids. In absence of external forces, it also conserves momentum at second-order and strictly conserves total energy at time $t^{n+1 / 2}$

$$
\begin{aligned}
& \boldsymbol{\mu}^{n+1 / 2}-\boldsymbol{\mu}^{n-1 / 2}=\boldsymbol{O}\left(\Delta x^{2}\right), \text { with } \boldsymbol{\mu}^{n+1 / 2}=\sum_{p} m_{p}^{n+1 / 2} \boldsymbol{u}_{p}^{n+1 / 2}, \\
& \mathcal{E}^{n+1 / 2}-\mathcal{E}^{n-1 / 2}=0, \text { with } \mathcal{E}^{n+1 / 2}=\sum_{p} \frac{1}{2} m_{p}^{n+1 / 2}\left(\boldsymbol{u}_{p}^{n+1 / 2}\right)^{2}+\sum_{c} m_{c} e_{c}^{n+1 / 2} .
\end{aligned}
$$

Demonstrations of Relations (42) and (43) can be found in [25]. Since the Lagrangian CSTS scheme of Subsection 3.1 is strictly conservative both in momentum and total energy [29], the residue in $\boldsymbol{O}\left(\Delta x^{2}\right)$ in (42) comes from the velocity correction of (24). This residue is second-order thanks to the Relation (27) except at the axis in axisymmetric geometries where it reduces to $O(\Delta x) \times O\left(\frac{\Delta r}{r}\right)$. Concerning the total energy, conservation of node (11) and centered (10) quantities during the remap together with our internal energy correction (25)-(26) proves the overall total energy conservation (43).

The Second Law of Thermodynamic is ensured and for isentropic problems the scheme also preserves entropy, at the scheme's order and for each material $\varphi[30]$

$$
\begin{aligned}
& s_{c}^{\varphi, n+1 / 2}-s_{c}^{\varphi, n-1 / 2}+O\left(\Delta x^{2}, \Delta t^{3}\right) \geq 0, \\
& s_{c}^{\varphi, n+1 / 2}-s_{c}^{\varphi, n-1 / 2}+O\left(\Delta x^{2}, \Delta t^{3}\right)+O\left(\Delta x^{2}\right)=0 .
\end{aligned}
$$

Relations (44) and (45) are proved in [25] using (29). The first error in $O\left(\Delta x^{2}, \Delta t^{3}\right)$ comes from the Lagrangian update of the internal energy which uses a second order quadrature rule for integrating the " $p d V$ " term in time. The second residue $O\left(\Delta x^{2}\right)$ in (45) is due to the variation of kinetic energy induced by the remap (see Subsection 2.5) and becomes $\boldsymbol{O}(\Delta x) \times O\left(\frac{\Delta r}{r}\right)$ in axisymmetric geometry. Note that it is absent from Inequality (44) because the per-cell kinetic energy discrepancy $\delta \mathcal{K}_{c}^{\varphi, \text { New }}$ is positive (29) up to round-off errors for each material $\varphi$.

Observe finally that the velocity correction (24) breaks the "DeBar condition" [4]: see discussion in Subsection 2.6 and Equation (27). A uniform velocity field is only preserved to the second-order-first-order in axisymmetric geometry on the axis $r=0$-in presence of smooth density gradients (second-order accuracy). The continuity with the multi-material Lagrangian CSTS scheme is ensured in the sense that no error is created in absence of rezoning since we have then $\lambda_{p}=1$.

\section{Numerical results}

\subsection{General numerical conditions}

First of all, pure remapping tests are considered in order to assess the properties of our remapping algorithm alone. To that end, the cyclic remapping test of [47] is re-used. Then, several classical—but demanding —-hydrodynamic test cases are performed to assess the built-in properties of our overall ALE CSTS algorithm: (i) second-order accuracy; (ii) conservation; (iii) thermodynamic consistency; and (iv) robustness. Three grid regularization strategies are used

- Euler: the grid is remapped at each time step onto a fixed cartesian grid, and the post remap velocity and internal energy corrections are performed;

- ALE: three iterations of the Jun's algorithm [46] are done at each time step for grid smoothing, and the post remap velocity and internal energy corrections are performed;

- Lagrange: no grid regularisation or remap are performed.

A classical and Space- and Time-Staggered non-conservative scheme—denoted as "STS" [5, 62] — will be used as a reference for robustness and will highlight the necessity of the total energy conservation. It is based on an "areaweighted" formulation of the momentum equation in axisymmetric geometry [5] and does not perform any velocity or energy correction in either the Lagrangian or the remapping phases. Unless otherwise specified, test cases are running with the following parameters: 
- Hydrodynamic time step respects the classical condition (39) with $\mathrm{CFL}=0.2$;

- The characteristic length is set to $\ell_{c}=\sqrt{A_{c}}$, where $A_{c}$ is the cell area;

- Dissipative terms are given in Appendix C.

All test cases are summarized in Tab. 2. In axisymmetric geometry, the $z$ and $r$ axes are respectively the horizontal and vertical axes. It is finally worth mentioning that the total energy conservation (43) and the kinetic energy discrepancy positivity (29) hold up to machine precision while total momentum is now conserved only to the second-order (42) for all test cases when corrections are activated. 


\begin{tabular}{|c|c|c|c|c|c|c|c|}
\hline $\begin{array}{l}\text { Test cases } \\
\text { References } \\
\text { Geometry }\end{array}$ & Initial conditions & Boundary conditions & $\begin{array}{l}\text { Adiabatic } \\
\text { constants }\end{array}$ & Dissipative terms & $\begin{array}{c}\text { Mesh size } \\
\text { Computational domain }\end{array}$ & Rezoning strategy & $\begin{array}{l}\text { Final time } \\
\text { CFL }\end{array}$ \\
\hline $\begin{array}{l}\text { Smooth Cyclic } \\
\text { remapping } \\
{[47]} \\
\text { plane }\end{array}$ & $\begin{array}{c}\rho_{0}=\sin (64 x y) \sin (64 x y) \\
\boldsymbol{u}_{0}=(\cos (8 x)+\sin (8 y), \cos (8 x)-\sin (8 y)) \\
e_{0}=\cos (10 x y) \cos (10 x y)\end{array}$ & I & I & I & $\begin{array}{c}32 \times 32,64 \times 64 \text { and } 128 \times 128 \text { cells } \\
{[0,1] \times[0,1]}\end{array}$ & $\begin{array}{l}\text { Non-orthogonal meshes } \\
\text { sequence [47] given by (46) }\end{array}$ & 1 \\
\hline $\begin{array}{c}\text { Cyclic } \\
\text { remapping } \\
{[47]} \\
\text { plane } \\
\end{array}$ & $\begin{array}{l}\rho_{0}=1, \boldsymbol{u}_{0}=(1,-0.3), e_{0}=0 \text { for } 0 \leq x \leq 0.5 \\
\rho_{0}=2, \boldsymbol{u}_{0}=(2,-0.6), e_{0}=1 \text { for } 0.5 \leq x \leq 1\end{array}$ & l & I & l & $\begin{array}{c}32 \times 32,64 \times 64 \text { and } 128 \times 128 \text { cells } \\
{[0,1] \times[0,1]}\end{array}$ & $\begin{array}{l}\text { Non-orthogonal meshes } \\
\text { sequence [47] given by (46) }\end{array}$ & 1 \\
\hline $\begin{array}{c}\text { Sod } \\
{[48]} \\
\text { axisymmetric } \\
\end{array}$ & $\begin{array}{c}\boldsymbol{u}_{0}=\mathbf{0} \\
\mathrm{Z} 1(z<0.5, r<0.5): \rho_{0}=1, p_{0}=1 \\
\mathrm{Z} 2(z \geq 0.5, r \geq 0.5): \rho_{0}=0.125, p_{0}=0.1 \\
\end{array}$ & $\begin{array}{c}\text { Left and Bottom: } \\
\boldsymbol{u} \cdot \boldsymbol{n}=0 \\
\text { Right and Top: } \boldsymbol{u}=\mathbf{0}\end{array}$ & $\begin{array}{l}\gamma_{\mathrm{Z} 1}=\frac{7}{5} \\
\gamma_{\mathrm{Z} 2}=\frac{7}{5}\end{array}$ & $\begin{array}{c}q_{1}=0.5 \\
q_{2}=(\gamma+1) / 4 \\
c_{\bar{\Phi}}=0 \\
\end{array}$ & $\begin{array}{l}55 \times 55 \text { cells } \\
{[0,1] \times[0,1]}\end{array}$ & $\begin{array}{c}\text { ALE \& Euler } \\
\text { rezoning and remap correction } \\
\text { at each time step } \\
\end{array}$ & $\begin{array}{l}0.2 \\
0.2\end{array}$ \\
\hline $\begin{array}{c}\text { Isentropic } \\
\text { vortex } \\
{[49-52]} \\
\text { plane }\end{array}$ & $\begin{array}{c}\rho_{0}=T_{0}^{1 /(\gamma-1)} \\
p_{0}=\rho_{0}^{\gamma}, \boldsymbol{u}_{0}=\delta \boldsymbol{u}_{0} \\
\delta \boldsymbol{u}_{0} \text { and } T_{0} \text { given by (47) and (48) }\end{array}$ & $u=0$ & $\frac{5}{3}$ & $\begin{aligned} q_{1} & =0 \\
q_{2} & =0 \\
c_{\bar{\Phi}} & =0\end{aligned}$ & $\begin{array}{c}50 \times 50,100 \times 100,200 \times 200 \text { and } \\
400 \times 400 \text { cells } \\
{[0,1] \times[0,1]}\end{array}$ & $\begin{array}{l}\text { ALE \& Euler } \\
\text { rezoning and remap correction } \\
\text { at each time step }\end{array}$ & $\begin{array}{c}1 \\
0.2\end{array}$ \\
\hline $\begin{array}{c}\text { Sedov } \\
{[53-55]} \\
\text { axisymmetric } \\
\end{array}$ & $\begin{array}{c}\rho_{0}=1, p_{0}=10^{-14} \\
\boldsymbol{u}_{0}=\mathbf{0} \\
\text { First cell: } \mathcal{E}_{0}=0.2468\end{array}$ & $\begin{array}{c}\text { Left and Bottom: } \\
\boldsymbol{u} \cdot \boldsymbol{n}=0 \\
\text { Right and Top: } \boldsymbol{u}=\mathbf{0}\end{array}$ & $\frac{5}{3}$ & $\begin{array}{c}q_{1}=0.5 \\
q_{2}=(\gamma+1) / 4 \\
c_{\bar{\Phi}}=1\end{array}$ & $\begin{array}{c}50 \times 50,100 \times 100 \text { and } 200 \times 200 \text { cells } \\
{[0,1.2] \times[0,1.2]}\end{array}$ & $\begin{array}{c}\text { ALE \& Euler } \\
\text { rezoning and remap correction } \\
\text { at each } 20 \text { time steps } \\
\end{array}$ & $\begin{array}{c}1 \\
0.2\end{array}$ \\
\hline $\begin{array}{l}\text { Triple point } \\
{[34,56,57]} \\
\text { axisymmetric }\end{array}$ & $\begin{array}{c}\boldsymbol{u}_{0}=\mathbf{0} \\
\mathrm{L}(z<1): \rho_{0}=1, p_{0}=1 \\
\operatorname{RT}(z>1, r>1.5): \rho_{0}=0.125, p_{0}=0.1 \\
\operatorname{RB}(z>1, r<1.5): \rho_{0}=1, p_{0}=0.1\end{array}$ & $\boldsymbol{u} \cdot \boldsymbol{n}=0$ & $\begin{aligned} \gamma_{\mathrm{L}} & =1.5 \\
\gamma_{\mathrm{RT}} & =1.5 \\
\gamma_{\mathrm{RB}} & =\frac{7}{5}\end{aligned}$ & $\begin{array}{c}q_{1}=0.5 \\
q_{2}=(\gamma+1) / 4 \\
\quad c_{\bar{\Phi}}=0\end{array}$ & $\begin{array}{c}140 \times 60 \text { and } 490 \times 210 \text { cells } \\
{[0,7] \times[0,3]}\end{array}$ & $\begin{array}{l}\text { ALE } \\
\text { rezoning and remap correction } \\
\text { at each time step }\end{array}$ & $\begin{array}{c}5 \\
0.2\end{array}$ \\
\hline $\begin{array}{c}\text { Shocked He } \\
\text { bubble } \\
{[58,59]} \\
\text { plane } \\
\end{array}$ & $\begin{array}{c}\boldsymbol{u}_{0}=\mathbf{0} \\
\text { He (disc): } \rho_{0}=1, p_{0}=10^{5} \\
\text { Air: } \rho_{0}=0.182, p_{0}=10^{5} \\
\text { Disc: } C=0.32 \boldsymbol{e}_{x}, R=0.025\end{array}$ & $\begin{array}{c}\text { Bottom and Top: } \\
\boldsymbol{u} \cdot \boldsymbol{n}=0 \\
\text { Left: } \boldsymbol{u}=\mathbf{0} \\
\text { Right: } \boldsymbol{u}=-124.824 \boldsymbol{e}_{x} \\
\end{array}$ & $\begin{aligned} \gamma_{\mathrm{He}} & =1.648 \\
\gamma_{\text {Air }} & =\frac{7}{5}\end{aligned}$ & $\begin{array}{c}q_{1}=0.5 \\
q_{2}=(\gamma+1) / 4 \\
c_{\bar{\Phi}}=0\end{array}$ & $\begin{array}{c}180 \times 50 \text { cells } \\
{[0,0.65] \times[-0.0445,0.0445]}\end{array}$ & $\begin{array}{l}\text { ALE } \\
\text { rezoning and remap correction } \\
\text { at each time step }\end{array}$ & $\begin{array}{l}134.2153 \times 10^{-6} \\
0.2\end{array}$ \\
\hline $\begin{array}{c}\text { Shocked He } \\
\text { bubble } \\
{[58,59]} \\
\text { axisymmetric } \\
\end{array}$ & $\begin{array}{c}\boldsymbol{u}_{0}=\mathbf{0} \\
\text { He }\left(\text { disc): } \rho_{0}=1, p_{0}=10^{5}\right. \\
\text { Air: } \rho_{0}=0.182, p_{0}=10^{5} \\
\text { Disc: } C=0.32 \boldsymbol{e}_{z}, R=0.0225\end{array}$ & $\begin{array}{c}\text { Bottom and Top: } \\
\boldsymbol{u} \cdot \boldsymbol{n}=0 \\
\text { Left: } \boldsymbol{u}=\mathbf{0} \\
\text { Right: } \boldsymbol{u}=-140.312 \boldsymbol{e}_{z} \\
\end{array}$ & $\begin{array}{c}\gamma_{\mathrm{He}}=1.648 \\
\gamma_{\mathrm{Air}}=\frac{7}{5}\end{array}$ & $\begin{array}{c}q_{1}=0.5 \\
q_{2}=(\gamma+1) / 4 \\
c_{\bar{\Phi}}=0\end{array}$ & $\begin{array}{c}520 \times 72 \text { cells } \\
{[0,0.65] \times[0,0.0445]}\end{array}$ & $\begin{array}{l}\text { ALE } \\
\text { rezoning and remap correction } \\
\text { at each time step }\end{array}$ & $\begin{array}{c}125.7463 \times 10^{-6} \\
0.2\end{array}$ \\
\hline
\end{tabular}

Table 2: Summary of test cases performed in this paper: coefficients $q_{1}$ and $q_{2}$ stand for respectively linear and quadratic terms of the artificial viscosity, coefficient $c_{\bar{\Phi}}$ controls the anti-hourglassing magnitude (see Appendix C). 


\subsection{Cyclic remapping}

We consider here two cyclic remapping tests [47] in order to assess the impact of the proposed corrections on the solution accuracy and monotonicity during remappings on time varying meshes. The first test remaps smooth fields, while the second one introduces a discontinuous internal energy. The mesh motion is given by a sequence of non-orthogonal meshes [47]:

$$
\begin{aligned}
& x(\xi, \eta, t)=\xi+\alpha(t) \sin (2 \pi \xi) \sin (2 \pi \eta), \\
& y(\xi, \eta, t)=\eta+\alpha(t) \sin (2 \pi \xi) \sin (2 \pi \eta), \\
& \alpha(t)= \begin{cases}t / 5 & \text { for } 0 \leq t \leq 0.5, \\
(1-t) / 5 & \text { for } 0.5 \leq t \leq 1,\end{cases}
\end{aligned}
$$

for $0 \leq \xi \leq 1,0 \leq \eta \leq 1$ sampled at $N$ points and $0 \leq t \leq 1$ sampled at $k_{\max }$ points. Fields are remapped on the unit square $[0,1]^{2}$ with the following sampling sequence $\left(N, k_{\max }\right)=(32,160),(64,320),(128,640)$ and $(256,1280)$. The most distorded $64 \times 64$ mesh during computation is displayed in Figure 3 .

Smooth fields. The following smooth fields

$$
\begin{aligned}
& \rho_{0}(x, y)=\sin \left(\frac{\pi}{2} x\right) \sin \left(\frac{\pi}{2} y\right), \\
& \boldsymbol{u}_{0}(x, y)=(\cos (8 x)+\sin (8 y), \cos (8 x)-\sin (8 y)), \\
& e_{0}(x, y)=\cos \left(\frac{\pi}{2} x\right) \cos \left(\frac{\pi}{2} y\right) .
\end{aligned}
$$

are successively remapped. The results of Figure 4 indicate a suboptimal order of convergence reduced to 1.3 and 1.46 without correction and as low as 0.66 after corrections. This loss of accuracy without correction is attributed to two factors: (i) the presence of distorded mesh during the computation on which the regularity assumption is not fullfilled-see Figure 3; and (ii) the effect of the first-order remap done at the boundaries. Moreover, although Equations (27) and (42) hold true, errors in $O\left(\Delta x^{2}\right)$ accumulate with time leading to a final convergence of order $O(\Delta x)$. In fact, the difference between the remapped velocity and the true velocity after one time step has a convergence order of around 1.3 , mainly due to errors at boundaries.

Non-smooth fields. Discontinuous fields taken from [47]

$$
\begin{aligned}
& \rho_{0}(x, y)= \begin{cases}2 & \text { for } y>(x-0.4) / 0.3, \\
1 & \text { for } y \leq(x-0.4) / 0.3,\end{cases} \\
& \boldsymbol{u}_{0}(x, y)= \begin{cases}(2,-0.6) & \text { for } y>(x-0.4) / 0.3, \\
(1, & -0.3) \text { for } y \leq(x-0.4) / 0.3,\end{cases} \\
& e_{0}(x, y)= \begin{cases}1 & \text { for } y>(x-0.4) / 0.3, \\
0 & \text { for } y \leq(x-0.4) / 0.3,\end{cases}
\end{aligned}
$$

are now successively remapped on the non-orthogonal meshes sequence. Isolines of velocity and internal energy are plotted both with and without corrections in Figure 5. It is no surprise that with corrections the monotonicity is lost both on velocity and internal energy. Errors around $1 \%$ to $3 \%$ on upper and lower bounds are observed for the $x$ component of the velocity while for internal energy, the lower bound is strictly respected and the upper bound has a variation of approximatively $2 \%$.

\subsection{Sod-like problem in axisymmetric geometry}

A Sod-like problem [48] in axisymmetric geometry is considered in this subsection. It corresponds to a two-states Riemann problem where the initial discontinuity is defined such that the three classical waves (expansion fan, contact discontinuity and shock) are: (i) planar along the axial direction $\boldsymbol{e}_{z}$; (ii) cylindrical along the radial direction $\boldsymbol{e}_{r}$; and (iii) spherical along the diagonal.

Figure 6 represents the final density map on an ALE $55 \times 55$ mesh. Density profiles on the $r=0$ axis are plotted in Figure 7 showing a good agreement with the analytical planar solution for all formulations. 


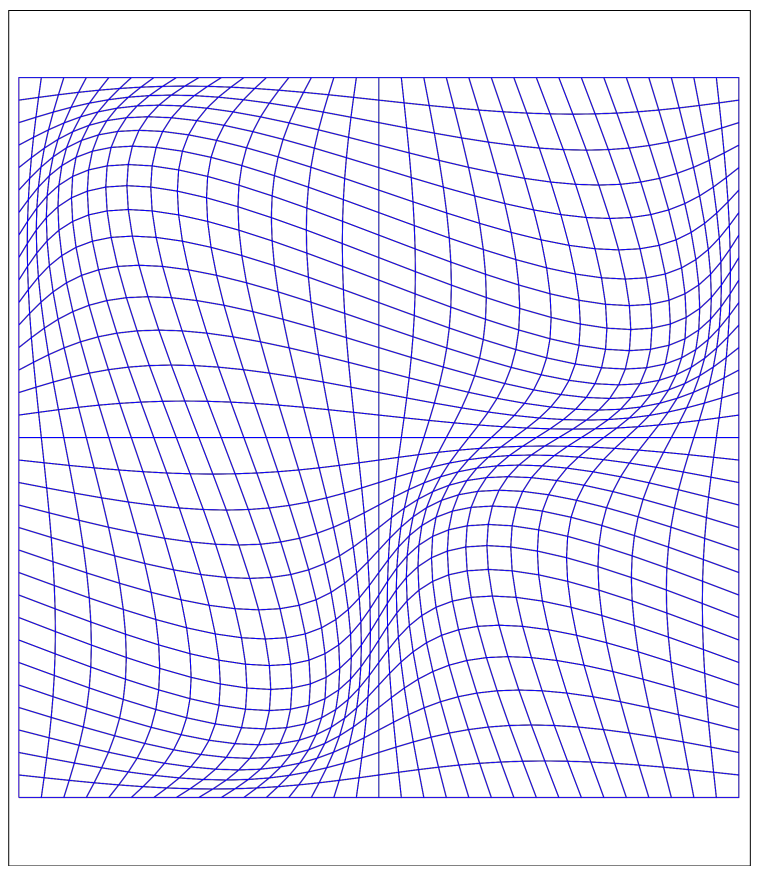

Figure 3: Cyclic remapping: most distorded mesh during the computation.

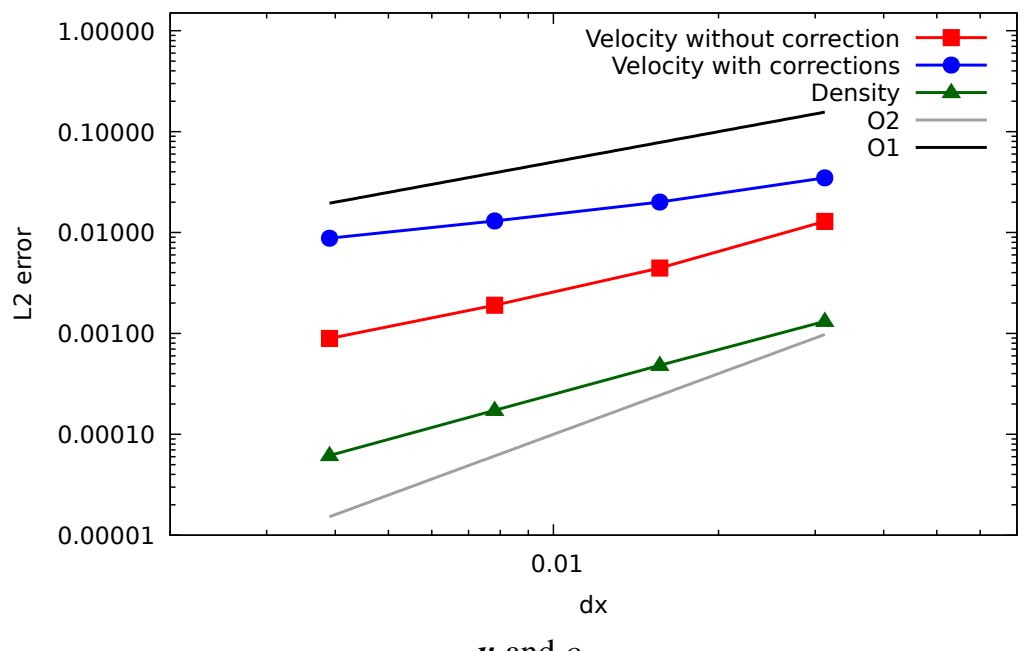

$\boldsymbol{u}$ and $\rho$

Figure 4: Cyclic remapping with smooth fields: $L^{2}$-error on the velocity with numerical orders estimated at 1.3 (without correction) and 0.66 (with corrections) and on the density (lower curve, not affected by correction step ) with numerical orders estimated at 1.5 . 


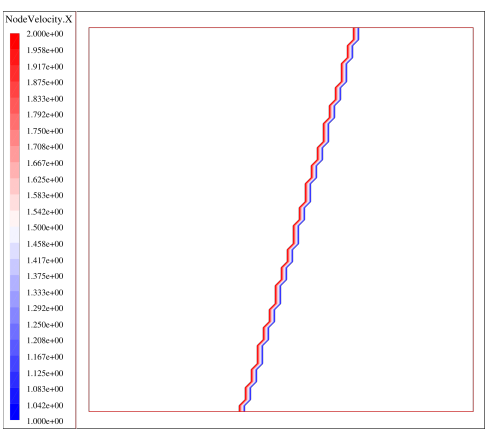

(a) $u_{x}$, ranging from 1.0 to 2.0

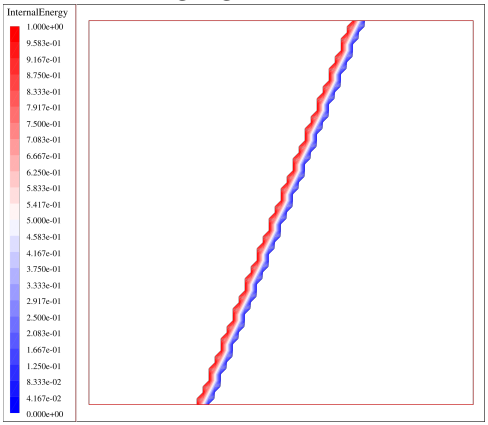

(d) $e$, ranging from 0.0 to 1.0

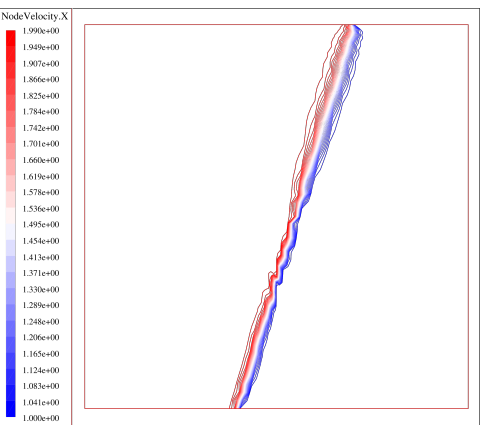

(b) $u_{x}$, ranging from 1.0 to 1.99

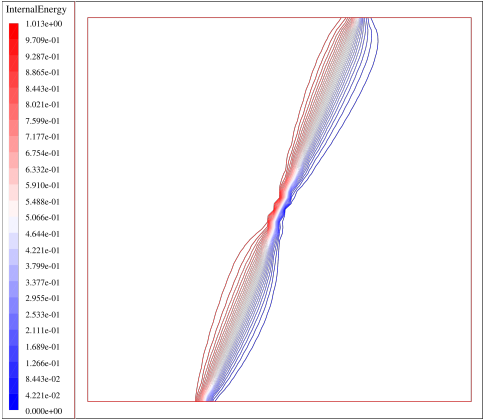

(e) $e$, ranging from 0.0 to 1.013

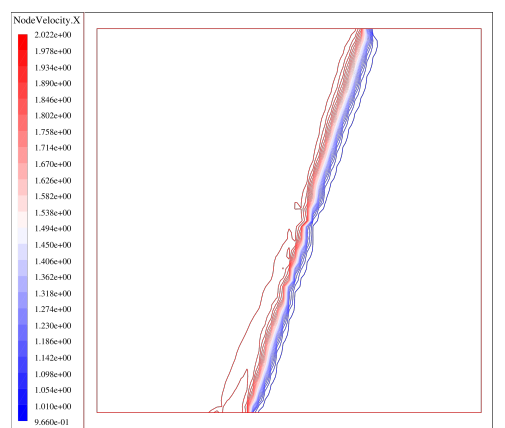

(c) $u_{x}$, ranging from 0.966 to 2.022

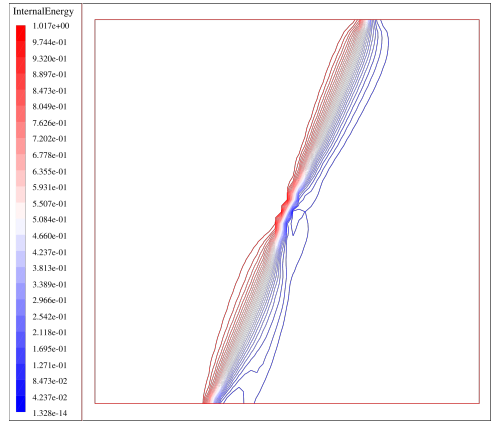

(f) $e$, ranging from 0.0 to 1.017

Figure 5: Cyclic remapping with non-smooth fields: isolines for $\left(N, k_{\max }\right)=(64,320)$ of (a) initial x-component of the velocity; (b) final xcomponent of the velocity without corrections; (c) final x-component of the velocity with corrections; (d) initial internal energy; (e) final internal energy without correction; and (f) final internal energy with corrections.

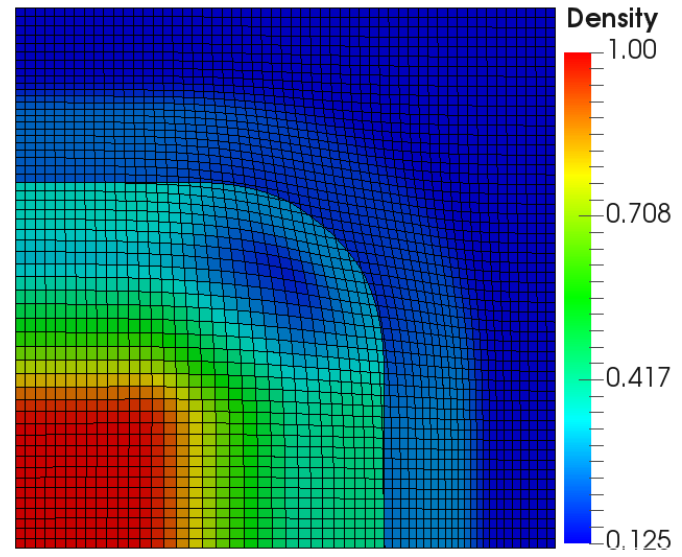

Figure 6: Sod-like problem in axisymmetric geometry: final density map on an ALE $55 \times 55$ mesh. 


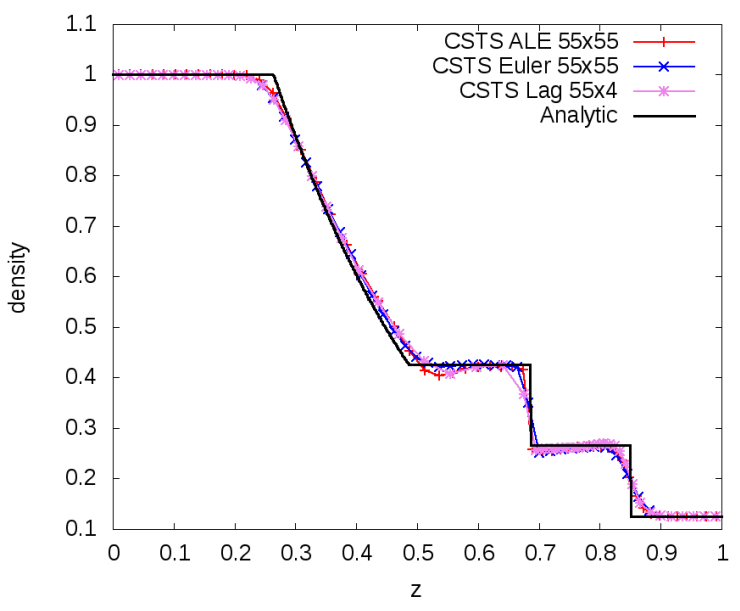

(a)

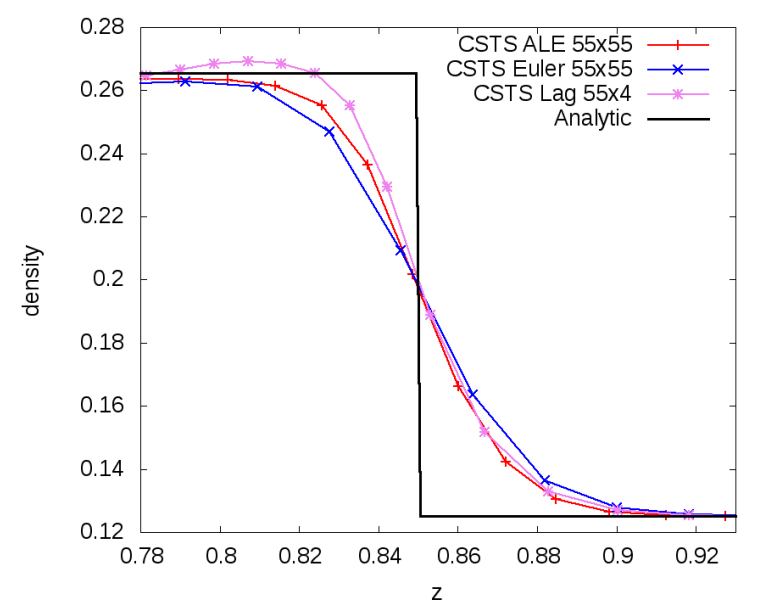

(b)

Figure 7: Sod-like problem in axisymmetric geometry. Density profiles on the axis $r=0$ for the CSTS scheme for Euler, ALE and Lagrangian runs: (a) global view; and (b) zoom around the shock.

\subsection{Isentropic vortex}

The isentropic vortex [49-52] is used here to assess the accuracy of our numerical scheme. A mean flow $\left(\bar{\rho}_{0}, \bar{p}_{0}, \overline{\boldsymbol{u}}_{0}\right)$ is perturbed in an isentropic way with $\left(\delta \rho_{0}, \delta p_{0}, \delta \boldsymbol{u}_{0}\right)$ such that

$$
\left\{\begin{array}{l}
\left(\bar{\rho}_{0}, \bar{p}_{0}, \overline{\boldsymbol{u}}_{0}\right)=(1,1, \mathbf{0}), \\
\delta \rho_{0}=T_{0}^{1 /(\gamma-1)}-1, \\
\delta p_{0}=T_{0}^{\gamma /(\gamma-1)}-1, \\
\delta u_{0, x}=\frac{\beta}{2 \pi} e^{\left(1-r^{2}\right) / 2}\left(y_{0}-y\right) \text { and } \delta u_{0, y}=\frac{\beta}{2 \pi} e^{\left(1-r^{2}\right) / 2}\left(x-x_{0}\right),
\end{array}\right.
$$

where $\left(x_{0}, y_{0}\right)=(5,0), r=\sqrt{\left(x-x_{0}\right)^{2}+\left(y-y_{0}\right)^{2}}$ and $\beta=5$ are respectively the vortex center, the vortex radius and the vortex strength. The initial temperature $T_{0}$ is given by

$$
T_{0}=1-\frac{(\gamma-1) \beta^{2}}{8 \gamma \pi^{2}} e^{1-r^{2}}
$$

The initial conditions (47) correspond to the condition $\frac{p}{\rho^{\gamma}}=\frac{p_{0}}{\rho_{0}^{\gamma}}=1$ associated to a perfect isentropic flow. Here, $L^{2}$-errors are computed for the density in order to assess the second-order accuracy and for the ratio $\frac{p}{\rho^{\gamma}}$ in order to measure the isentropic property of the numerical solution. Figure 8 displays the final ALE $50 \times 50$ mesh: all quantities vanish away from the center $\left(x_{0}, y_{0}\right)$-including the velocity — to avoid the first-order remap at boundaries. Figure 9 shows a second-order accuracy both on density and entropy for ALE and Euler simulations. Exception is the velocity where an order of around 1.2 is observed. As for the cyclic remapping, second-order errors $O\left(\Delta x^{2}\right)$ coming from (27) accumulate during time causing a drop of almost one order on the convergence.

Figure 10 illustrates the contribution of the internal energy gradient (15) on the numerical isentropy compared to a monotone gradient limited with the Van Leer method [32]. While the improvement on the numerical isentropy is marginal in the ALE formulation (see Figure 10(a)), it has a significant impact for the Euler case (see Figure 10(b)): we obtain a gain of one order on the numerical isentropy and the ratio $\frac{p}{\rho^{\gamma}}$ error is ten times lower on the $400 \times 400$ grid when the isentropic internal energy gradient (15) is used.

\subsection{Spherical Sedov blast wave}

The spherical Sedov blast wave [53] corresponds to an expanding, infinitely strong shock generated by an instantaneous point source of energy and propagating in a cold perfect gas of initial pressure $p_{0}=10^{-14}$. The value of the initial total energy $E_{\text {blast }}$ - depending on the geometry and the initial density distribution [54, 55]—is chosen such that 


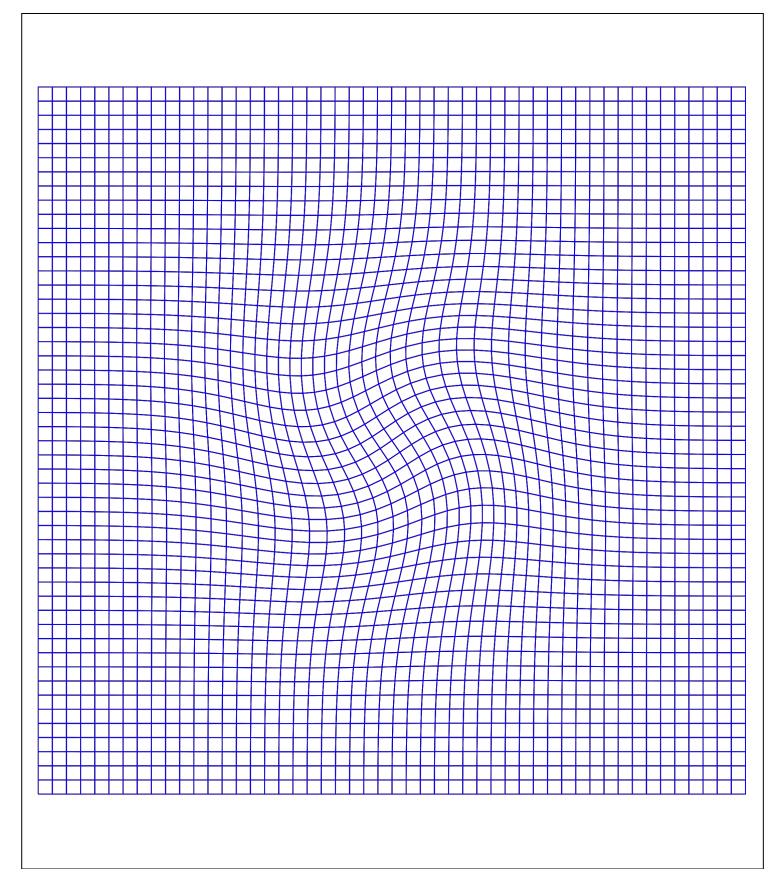

Figure 8: Isentropic vortex: final ALE $50 \times 50$ mesh.

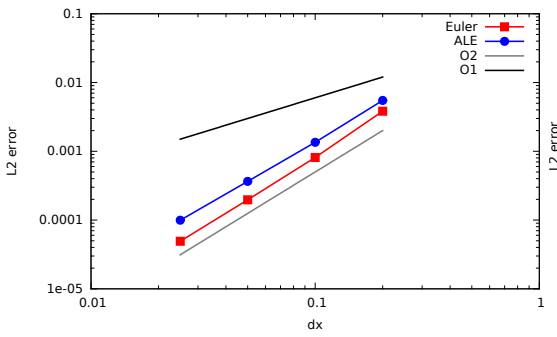

(a) $\rho$

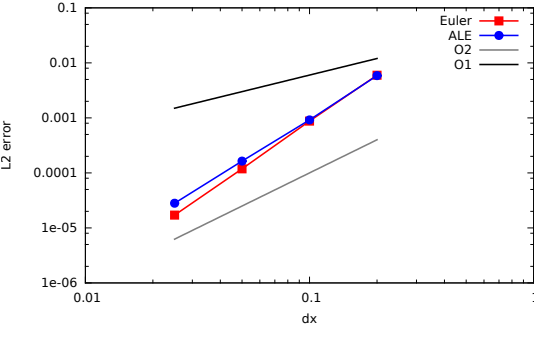

(b) $\frac{p}{\rho^{\gamma}}$

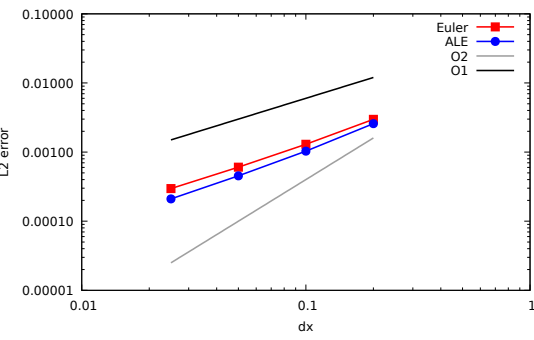

(c) $u$

Figure 9: Isentropic vortex with correction: $L^{2}$-errors both for the ALE and Euler simulations on (a) the density $\rho$ with numerical orders estimated at 2.0 (Euler) and at 1.8 (ALE); (b) the ratio $\frac{p}{\rho^{\gamma}}$ with numerical orders estimated at 2.8 (Euler) and 2.6 (ALE); and (c) the velocity $u$ with numerical orders estimated at 1.1 (Euler) and 1.2 (ALE). Because of a more regular mesh, the decrease in convergence due to velocity correction is less pronounced than in the cyclic remapping test. 


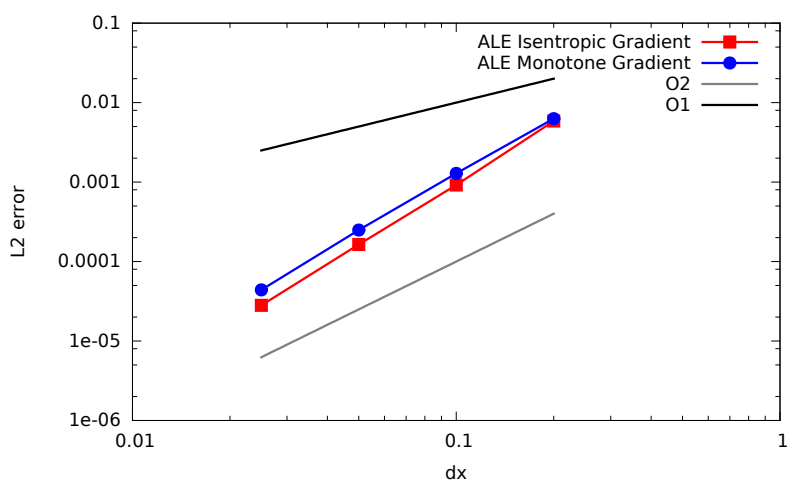

(a) ALE

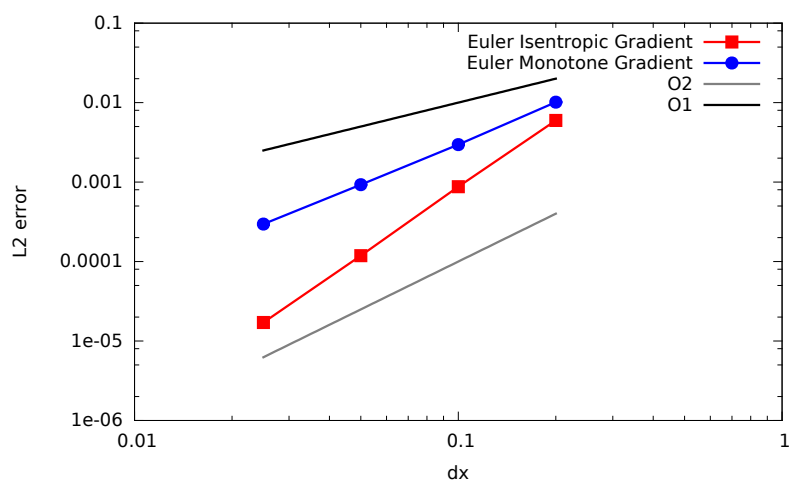

(b) Euler

Figure 10: Isentropic vortex: $L^{2}$-errors on the ratio $\frac{p}{\rho^{\gamma}}$ both for a classical limited internal energy gradient and for the isentropic internal energy gradient (15): (a) ALE with an estimated order around 2.4 when the gradient is limited; and (b) Euler with an estimated order around 1.7 when the gradient is limited.

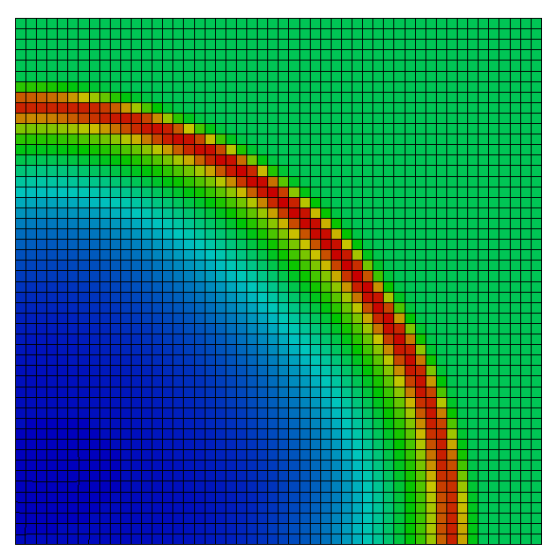

(a) Euler

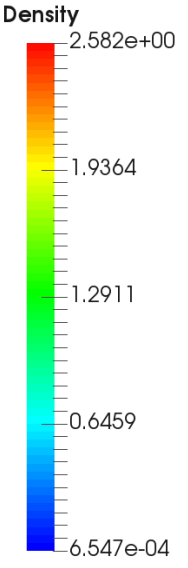

.547e-04

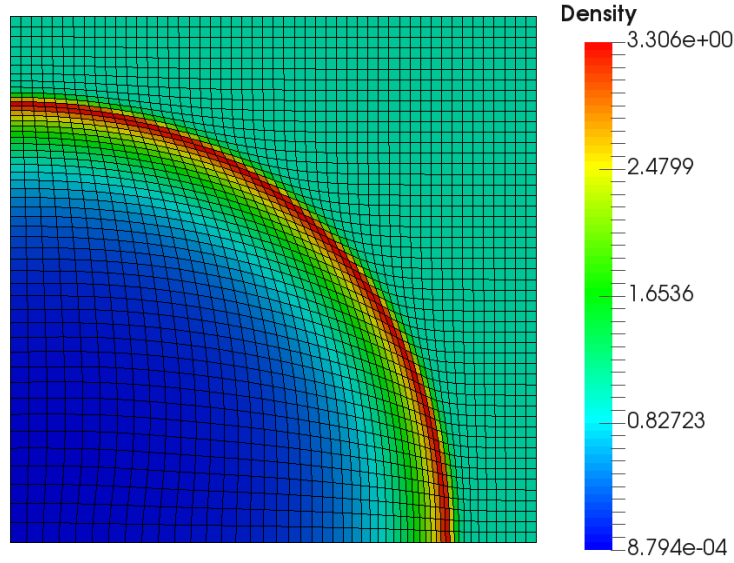

(b) ALE

Figure 11: Spherical Sedov blast wave: final density maps on $50 \times 50$ meshes for (a) Euler and (b) ALE simulations.

the shock wave reaches the position $r_{\text {shock }}=1$ at the final time $t=1$. This test case is particularly sensitive to total energy discrepancies and is thus often used to assess the strict total energy conservation.

For this case the rezoning step is performed every 20 time steps in order to obtain a quasi-Lagrangian mesh on the shock, thus requiring an anti-hourglassing method. Figure 11 displays final density maps on $50 \times 50$ meshes and compares the Eulerian and the ALE formulations. The quasi-Lagrangian mesh motion on the shock wave clearly allows a better capture of the analytic density peak $\rho_{1}=4$ for the ALE formulation. Figure 12(b) shows that our algorithm conserves total energy up to machine precision. When the mesh is refined, we observe that our simulations converge to the correct value (see Figure 12(c)) while the non-conservative STS scheme produces an error around $1 \%$ on the analytical shock position $r_{\text {shock }}=1$ for the $200 \times 200$ grid (see Figure $12(\mathrm{a})$ ).

\subsection{Axisymmetric multi-material triple point problem}

The triple point problem is a two-material, three-states Riemann problem where two shock waves propagate at different velocities in two different materials along the horizontal contact discontinuity generating a vortex at the triple point.This test case is usually performed in plane geometry [34, 56]. Axisymmetric results are less frequent but can be found in e.g. [57]. Initial and boundary conditions are given in Tab. 2 and are identical to those described in [57]. An ALE mesh motion is here necessary since a Lagrangian computation fails to reach the final time $t=5$ because of the strong mesh tangling in the vortex area [60]. 


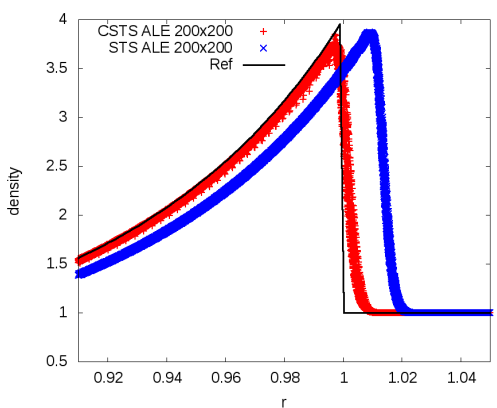

(a)

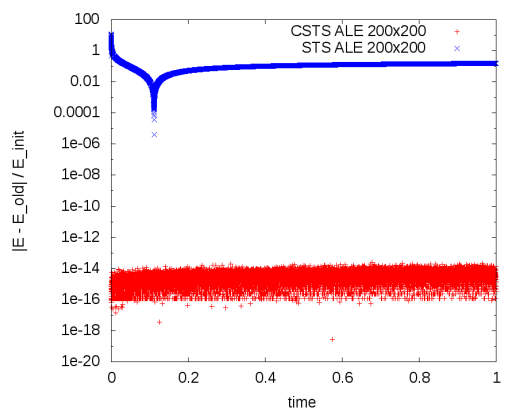

(b)

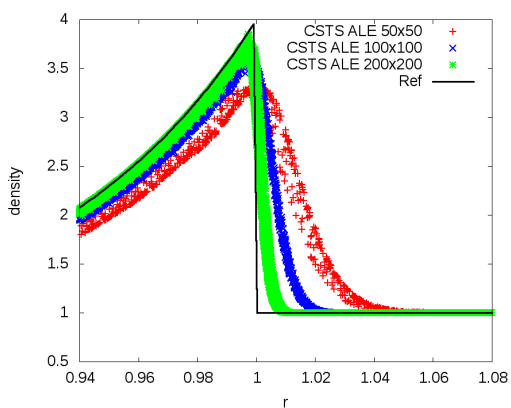

(c)

Figure 12: Spherical Sedov blast wave: (a) radial density profiles for the CSTS and STS schemes on a $200 \times 200$ ALE grid and for the reference solution [54, 55]; (b) relative total energy variation $\left|\mathcal{E}^{n+1 / 2}-\mathcal{E}^{n-1 / 2}\right| / \mathcal{E}^{0}$ defined in (43) as a function of time for the CSTS and STS schemes on a $200 \times 200$ ALE grid; and (c) radial density profiles under mesh refinement, superposed with the reference solution, for the CSTS scheme with an ALE mesh motion.

Figure 13 displays the final internal energy map on an ALE $140 \times 60$ mesh. The material positions and the overall behavior of the solution are consistent with the Eulerian numerical results shown in [57]. The main difference concerns the vortex capture for which a smoother result - and thus a better roll-up capture-is shown in [57] due to a higher numerical dissipation provided by the solver and the Eulerian mesh motion. This test case possesses no analytical solution nor converged solution [16] and it is mainly used here as a robustness test. Kelvin-Helmhotz-like instabilities grow without any control under mesh refinement-except from the numerical dissipation which is strongly dependent on the numerical solver-because of the absence of a physical viscosity term in the Euler's equations. Observe that in this case, the algorithm becomes unstable and explodes numerically before the final time if we correct the internal energy after remap without correcting the velocity.

\subsection{Shocked Helium bubble}

We consider here the numerical simulation of a shocked Helium bubble in Air. Experimental results are available in $[58,59]$ both for plane and axisymmetric geometries. Numerical results are also provided in [2, 61]. Initial and boundary conditions are identical to those described in [57].

\subsubsection{Plane geometry}

The domain $[0,0.65] \times[-0.0445,0.0445]$ is filled with Air at room pressure, except in a disc centered at $C=$ $0.32 \boldsymbol{e}_{x}$ of radius $R=0.025$ which is filled with Helium. A piston at velocity $u^{\star} \boldsymbol{e}_{x}$-where $u^{\star}=-124.824$ is computed thanks to Rankine-Hugoniot relations — on the right boundary generated a Mach 1.22 shock, hitting the bubble at $t_{i}=668.153 \times 10^{-6}$. The computation is running until the final time $t=t_{i}+674 \times 10^{-6}$.

Figure 14 shows the density map at different time $t_{s}$ after the shock-bubble interaction on a $180 \times 50$ ALE mesh. The bubble shape is compared to the STS scheme and the experimental result of [58] in Figure 15. The bubble shape provided by the CSTS scheme matches with the experimental result while the STS scheme produces a result closer to those found in [2, 61], obtained on a finer grid. As mentioned in [61] and in Subsection 4.6, the lack of physical dissipation explains the differences with experiments. However, remapping on the coarse mesh produces enough numerical dissipation to be comparable with experiments for the CSTS scheme.

\subsubsection{Axisymmetric geometry}

The shock-bubble interaction is now computed on the domain $[0,0.65] \times[0,0.0445]$. Helium bubble, surrounded by Air, is centered at $C=0.32 \boldsymbol{e}_{z}$ with a radius of $R=0.0225$. The shock is still generated by a piston of velocity $u^{\star} \boldsymbol{e}_{z}$ with $u^{\star}=-140.312$ given by Rankine-Hugoniot relations to have a 1.25 Mach shock. The final time is $t=$ $t_{i}+600 \times 10^{-6}$, where $t_{i}=657.463 \times 10^{-6}$ still corresponds to the interaction time between the shock and the bubble.

Numerical results on a $520 \times 72$ ALE mesh are displayed in Figures 16 and 17. While instabilites affect the final bubble shape, Figure 17 shows a good agreement with the experimental result, especially concerning the roll-up capture at the rear of the bubble for the CSTS scheme. These instabilities can be damped with a coarser grid but the lack of cells prevents both the STS and CSTS schemes from capturing the bubble structures. 


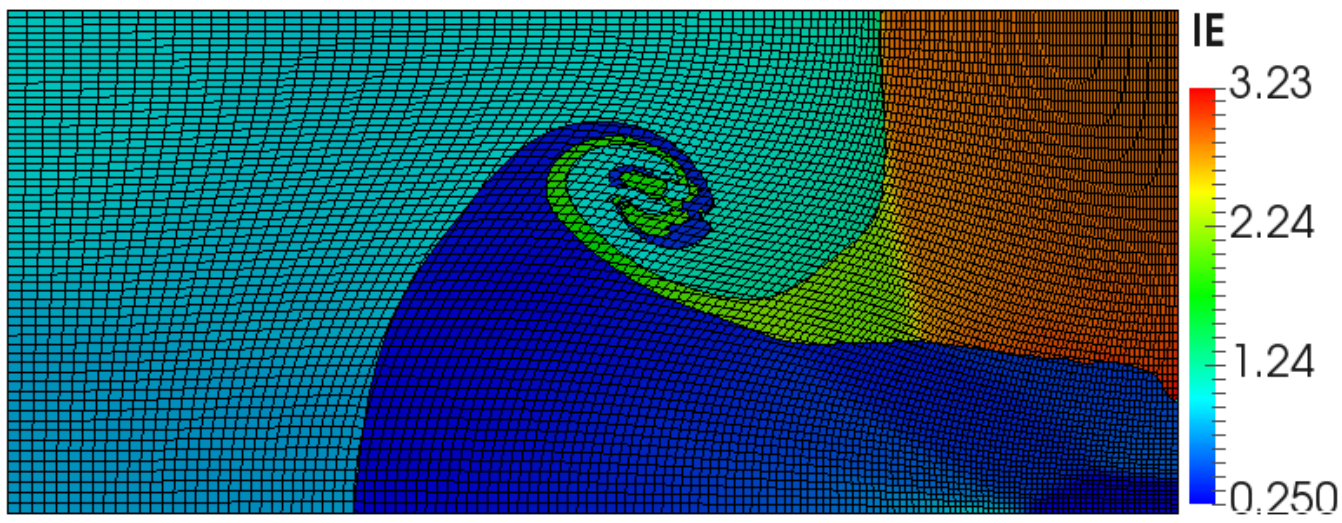

(a) $140 \times 60$ ALE mesh

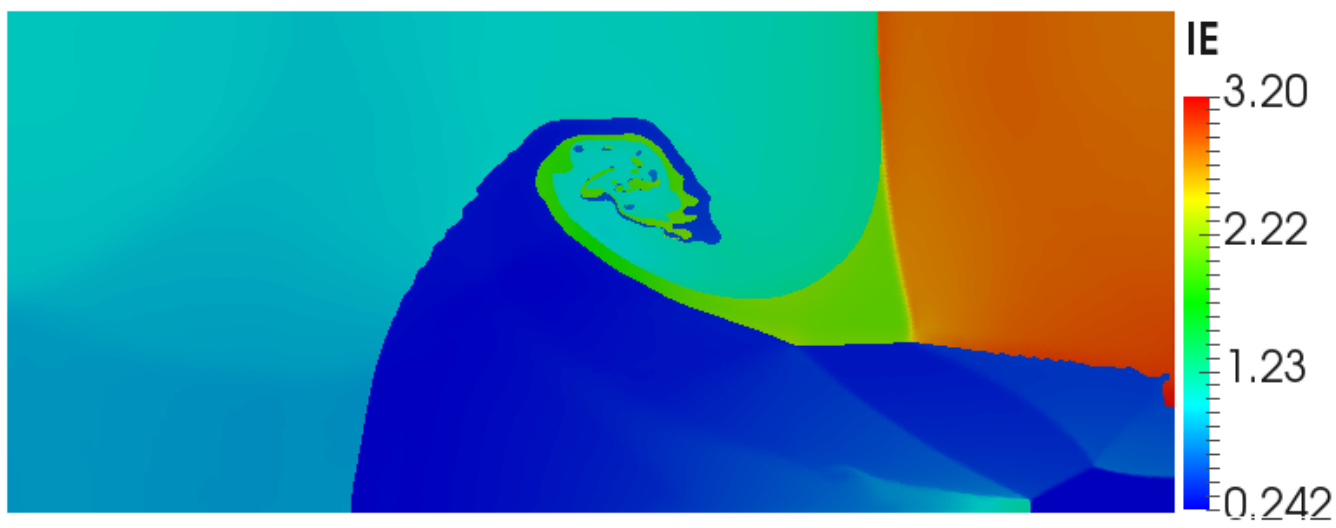

(b) $490 \times 210$ ALE mesh

Figure 13: Axisymmetric triple point problem: final internal energy maps obtained on (a) a 140×60 ALE grid (with representation of the underlying mesh) and (b) on a $490 \times 210$ mesh. 


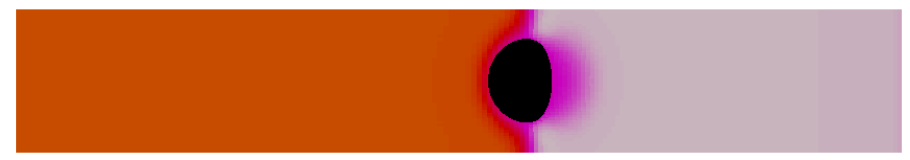

(a) $t_{s}=32.127 \times 10^{-6}$

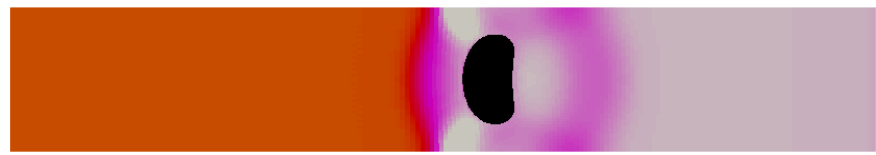

(b) $t_{s}=132.135 \times 10^{-6}$

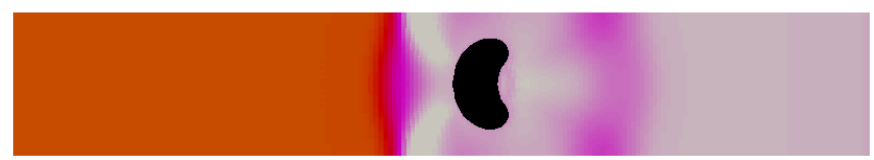

(c) $t_{s}=182.058 \times 10^{-6}$

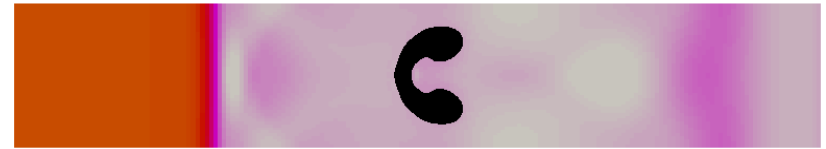

(d) $t_{s}=482.227 \times 10^{-6}$

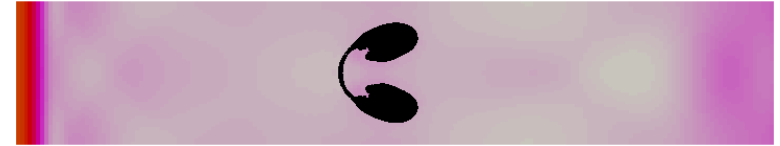

(e) $t_{s}=674 \times 10^{-6}$

Density

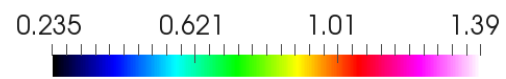

Figure 14: Cylindrical shocked Helium bubble: density maps on a $180 \times 50$ ALE mesh for the CSTS scheme at times (a) $t_{s}=32.127 \times 10^{-6}$; (b) $t_{s}=132.135 \times 10^{-6}$; (c) $t_{s}=182.058 \times 10^{-6} ;$ (d) $t_{s}=482.227 \times 10^{-6}$; and (e) $t_{s}=674 \times 10^{-6}$. Time $t_{s}$ corresponds to the elapsed time after the shock-bubble interaction time $t_{i}$.

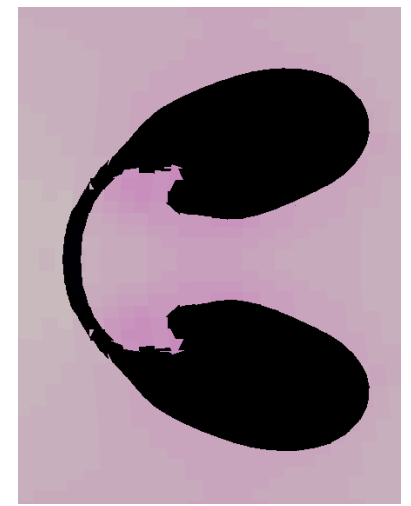

(a) CSTS

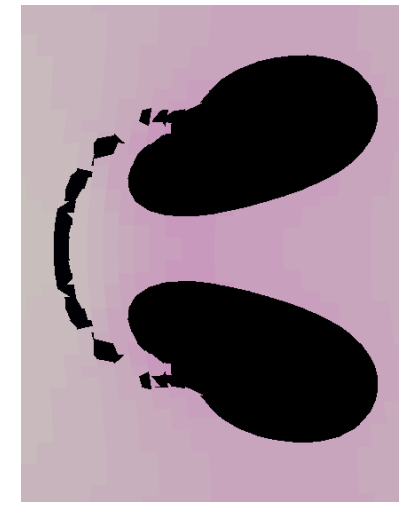

(b) STS

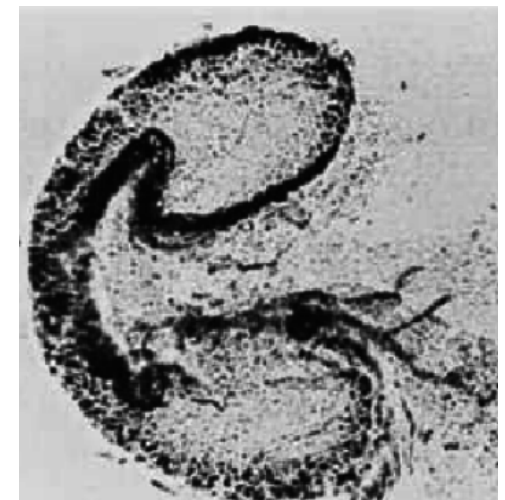

(c) Schlieren diagram

Figure 15: Cylindrical shocked Helium bubble: zoom on the material interface on a $180 \times 50$ ALE mesh for (a) the CSTS scheme (b) the STS scheme and (c) for the Schlieren diagram [58]. 


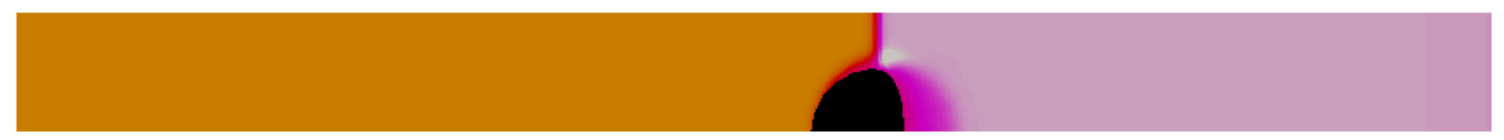

(a) $t_{s}=42.636 \times 10^{-6}$

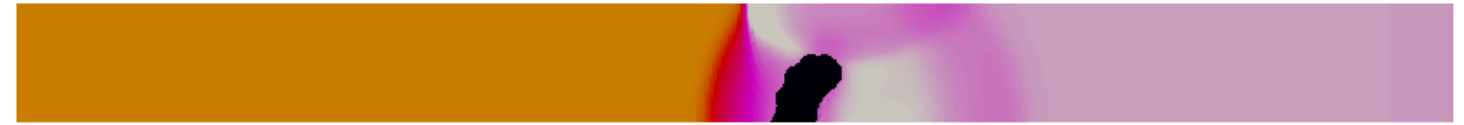

(b) $t_{s}=142.589 \times 10^{-6}$

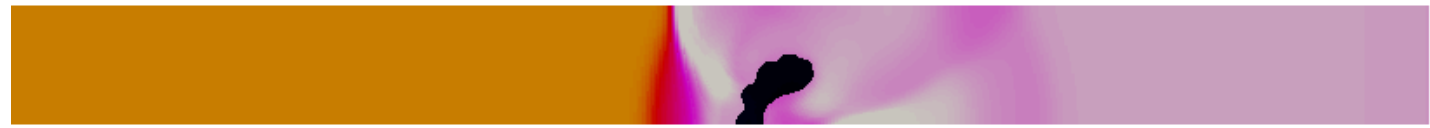

(c) $t_{s}=192.543 \times 10^{-6}$

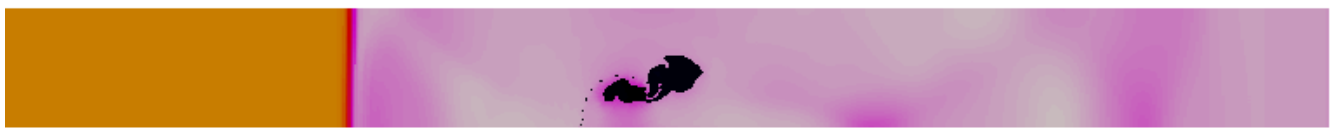

(d) $t_{s}=442.577 \times 10^{-6}$

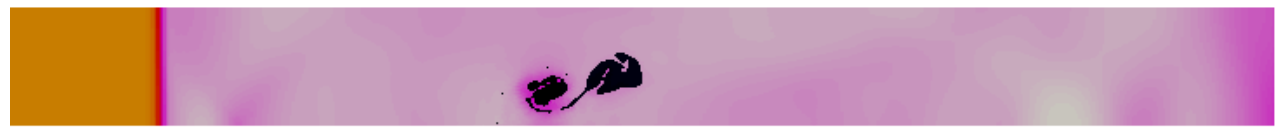

(e) $t_{s}=600 \times 10^{-6}$

Density

$\begin{array}{llll}0.230 & 0.643 \quad 1.05 & 1.47\end{array}$

Figure 16: Spherical shocked Helium bubble: density maps on a $520 \times 72$ ALE mesh for the CSTS scheme at times (a) $t_{s}=42.636 \times 10^{-6}$; (b) $t_{s}=142.589 \times 10^{-6}$; (c) $t_{s}=192.543 \times 10^{-6}$; (d) $t_{s}=442.577 \times 10^{-6}$; and (e) $t_{s}=600 \times 10^{-6}$.

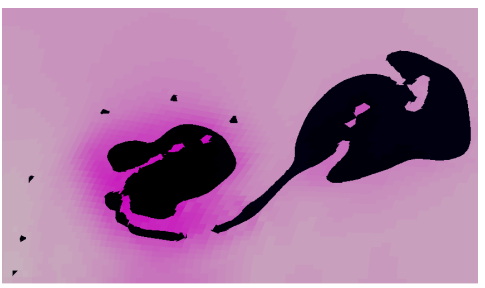

(a) CSTS

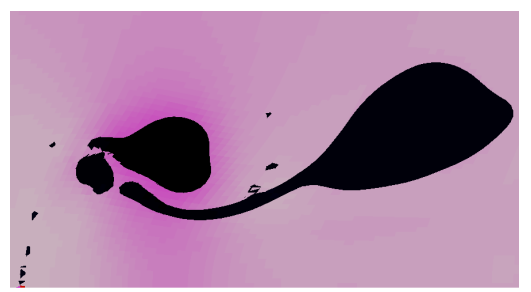

(b) STS

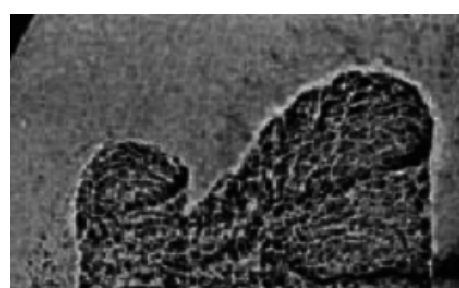

(c) Schlieren diagram

Figure 17: Spherical shocked Helium bubble: zoom on the material interface on a $520 \times 72$ ALE mesh for (a) the CSTS scheme (b) the STS scheme and (c) for the Schlieren diagram [58]. 


\section{Conclusions}

This paper presents a simple attempt to extend entropic numerical space-staggered schemes to the ALE methodology. For that purpose, a conservative and thermodynamically consistent staggered-remapping method is developed. This is achieved through instantaneous corrections, at a low computational cost, both of velocity and internal energy. The continuity with the Lagrangian step is preserved. However, the algorithm analysis shows that the strict momentum conservation is lost-although it is still conserved to the scheme's order-as well as some other classical remapping properties: "DeBar consistency" [4] and monotonicity. The remapping method is then complemented with the Lagrangian CSTS scheme of [24]. We emphasize the link between instantaneous corrections of Subsection 2.6 and the modification of momentum fluxes of [24]. They have the same role respectively in the remapping and the Lagrangian steps: local corrections on linear momentum are introduced in order to be conservative and to control entropy simultaneously. Several classical—but demanding—-test cases are performed to assess the properties of the whole ALE algorithm.

In a close future, different formulations of artificial viscosity will be tested. Some considerations on the symmetry preservation are also planed by considering an interpolation-based method to remap the velocity, instead of the classical intersection-based remap. Let us note that this is also better in terms of computational cost. Finally, the ALE algorithm will be coupled with elastic-plastic behavior. Space-staggered discretizations are well suited for solids since it allows to accurately compute the stress. Therefore, following the hypoelasticity framework [5], $\tau^{\varphi}$ can be easily computed at half time $t^{n+1 / 2}$ before being remapped.

\section{Acknowledgements}

We are grateful towards A. Llor for helpful discussions and for providing the isentropic gradient for the internal energy remap and to J.P. Perlat for his permanent expertise and help.

\section{Appendix A. Isentropic gradient construction for internal energy}

Starting from the Gibbs relation $d e=-p d v+T d s$, one gets $\boldsymbol{\nabla} e=-p \nabla v+T \nabla s$, where $v=\frac{1}{\rho}$ is the specific volume. Working with the density $\rho$ instead of the specific volume $v$ and making the assumption that $\boldsymbol{\nabla} s=\mathbf{0}$, the previous relation becomes $\boldsymbol{\nabla} e=-p \boldsymbol{\nabla} v=\frac{p}{\rho^{2}} \boldsymbol{\nabla} \rho$. We finally multiply by the density $\rho$ to get $\rho \boldsymbol{\nabla} e=\frac{p}{\rho} \boldsymbol{\nabla} \rho$ and we add $e \nabla \rho$

$$
\boldsymbol{\nabla}(\rho e)=\rho \boldsymbol{\nabla} e+e \boldsymbol{\nabla} \rho=\frac{p}{\rho} \boldsymbol{\nabla} \rho+e \boldsymbol{\nabla} \rho=\left(\frac{p}{\rho}+e\right) \boldsymbol{\nabla} \rho .
$$

\section{Appendix B. Analysis of the mass transfer error}

Our objective in this appendix is to prove that in axisymmetric geometry, on a regular mesh, and when corner masses $m_{c p}^{\mathrm{New}}$ and node masses $m_{p}^{\mathrm{New}}$ are built by (30), the mass transfer satisfies

$$
\begin{aligned}
& m_{p}^{\mathrm{New}}-m_{p}^{\mathrm{R}}=m_{p}^{\mathrm{R}} \times O(\Delta x) \times O\left(\frac{\Delta r}{r}\right), \\
& \mathcal{K}_{p}^{\mathrm{New}}-\frac{1}{2} m_{p}^{\mathrm{R}}\left(\boldsymbol{u}_{p}^{\mathrm{R}}\right)^{2}=\mathcal{K}_{p}^{\mathrm{New}} \times O(\Delta x) \times O\left(\frac{\Delta r}{r}\right) .
\end{aligned}
$$

To simplify notations, we omit below the index "New" since all quantities are constructed on the regularized mesh.

On a regular mesh, the different sub-volumes introduced in the remap and in (30) satisfy

$$
\begin{aligned}
& \frac{V_{c p}}{V_{c}}=\frac{1}{4}+O(\Delta x) \times O\left(\frac{\Delta r}{r}\right), \\
& \frac{V_{c p}^{M}}{V_{c}}=\frac{1}{4}+O(\Delta x) \times O\left(\frac{\Delta r}{r}\right),
\end{aligned}
$$

where $V_{c p}=V_{p} \cap V_{c}$ denotes the intersection of the dual cell volume $V_{p}$ used in the remapping step with the cell volume $V_{c}$. If we introduce $\beta_{c p}=\frac{2}{3} \frac{V_{c p}^{\mathrm{M}}}{V_{c}}+\frac{1}{3} \frac{V_{c p}^{\mathrm{W}}}{V_{c}}$ and $V_{p}^{\text {mass }}=\sum_{c \in C(p)} \beta_{c p} V_{c}$, we deduce from (B.3) that we have

$$
\frac{V_{p}^{\text {mass }}}{V_{p}}=1+O(\Delta x) \times O\left(\frac{\Delta r}{r}\right) .
$$


By construction of the node mass in the Lagrangian step, we have

$$
m_{p}=\sum_{c \in C(p)} \beta_{c p} \int_{V_{c}} \tilde{\rho}_{1}(\boldsymbol{x}) r d r d z
$$

where $\tilde{\rho}_{1}(\boldsymbol{x})$ is the density field reconstructed from cell-centered values $\left\{\rho_{c}\right\}$ on the Lagrangian mesh. Since this field $\tilde{\rho}_{1}(\boldsymbol{x})$ is a second-order reconstruction of the solution $\rho$, we have

$$
\begin{aligned}
& m_{p}= \sum_{c \in C(p)} \beta_{c p} \int_{V_{c}} \rho(\boldsymbol{x}) r d r d z+V_{p}^{\text {mass }} \times O\left(\Delta x^{2}\right) \\
&= \sum_{c \in C(p)} \beta_{c p} \int_{V_{c}}\left[\rho\left(\boldsymbol{x}_{p}\right)+(\boldsymbol{\nabla} \rho)_{p} \cdot\left(\boldsymbol{x}-\boldsymbol{x}_{p}\right)\right]\left[\left(r-r_{p}\right)+r_{p}\right] d r d z \\
&+V_{p}^{\text {mass }} \times O\left(\Delta x^{2}\right)
\end{aligned}
$$

On a regular mesh, the node $\boldsymbol{x}_{p}$ is close to the $2 \mathrm{D}$ geometric center of $\cup_{c \in C(p)} V_{c}$, and hence term A is high-order. From (B.3) we have that term B is of order $V_{p}^{\text {mass }} \times O\left(\Delta x^{2}\right) \times O\left(\frac{\Delta r}{r}\right)$. And last, term $\mathrm{C}$ writes

$$
\begin{aligned}
\sum_{c \in C(p)} \beta_{c p} \int_{V_{c}}(\nabla \rho)_{p} \cdot\left(\boldsymbol{x}-\boldsymbol{x}_{p}\right)\left(r-r_{p}\right) d r d z & =\sum_{c \in C(p)} \beta_{c p} \int_{V_{c}}(\boldsymbol{\nabla} \rho)_{p} \cdot\left(\boldsymbol{x}-\boldsymbol{x}_{p}\right) \frac{r-r_{p}}{r} r d r d z \\
& =\left(\sum_{c \in C(p)} \beta_{c p} V_{c}\right) \times O(\Delta x) \times O\left(\frac{\Delta r}{r}\right) \\
& =V_{p}^{\text {mass }} \times O(\Delta x) \times O\left(\frac{\Delta r}{r}\right) .
\end{aligned}
$$

Altogether, we get

$$
m_{p}=\left(\sum_{c \in C(p)} \beta_{c p} V_{c}\right) \rho\left(\boldsymbol{x}_{p}\right)+V_{p}^{\mathrm{mass}} \times O(\Delta x) \times O\left(\frac{\Delta r}{r}\right)
$$

If $\tilde{\rho}_{2}(\boldsymbol{x})$ now denotes the density field reconstructed from node-centered values $\left\{\rho_{p}\right\}$ with $\rho_{p}$ defined by (16), we have

$$
\tilde{\rho}_{2}(\boldsymbol{x})=\rho(\boldsymbol{x})\left(1+O(\Delta x) \times O\left(\frac{\Delta r}{r}\right)\right) .
$$

Then, a direct calculation yields

$$
\begin{aligned}
m_{p}^{\mathrm{R}} & =\int_{V_{p}} \tilde{\rho}_{2}(\boldsymbol{x}) r d r d z \\
& =\int_{V_{p}} \rho(\boldsymbol{x}) r d r d z+V_{p} \times O(\Delta x) \times O\left(\frac{\Delta r}{r}\right)
\end{aligned}
$$




$$
\begin{aligned}
=V_{p} \rho\left(\boldsymbol{x}_{p}\right)+\int_{V_{p}}(\boldsymbol{\nabla} \rho)_{p} \cdot\left(\boldsymbol{x}-\boldsymbol{x}_{p}\right) r_{p} d r d z \\
\quad+\int_{V_{p}}(\boldsymbol{\nabla} \rho)_{p} \cdot\left(\boldsymbol{x}-\boldsymbol{x}_{p}\right)\left(r-r_{p}\right) d r d z+V_{p} \times O(\Delta x) \times O\left(\frac{\Delta r}{r}\right) .
\end{aligned}
$$

We thus get

$$
m_{p}^{\mathrm{R}}=V_{p} \rho\left(\boldsymbol{x}_{p}\right)+V_{p} \times O(\Delta x) \times O\left(\frac{\Delta r}{r}\right) .
$$

By combining (B.5), (B.7) and (B.4), we deduce (B.1)

$$
m_{p}-m_{p}^{\mathrm{R}}=V_{p} \times O(\Delta x) \times O\left(\frac{\Delta r}{r}\right) .
$$

The same demonstration is valid for (B.2). We write the difference in (B.2) under the same integral using the kinetic energy field $\tilde{\mathcal{K}}$ reconstructed from node values $\left\{\mathcal{K}_{p}\right\}$ —defined in (16)—on the Lagrangian mesh and the Definition (17) of $m_{p}^{\mathrm{R}}$ to get

$$
\mathcal{K}_{p}-\frac{1}{2} m_{p}^{\mathrm{R}}\left(\boldsymbol{u}_{p}^{\mathrm{R}}\right)^{2}=\int_{V_{p}}\left[\tilde{\mathcal{K}}(\boldsymbol{x})-\frac{1}{2} \tilde{\rho}_{2}(\boldsymbol{x})\left(\boldsymbol{u}_{p}^{\mathrm{R}}\right)^{2}\right] r d r d z=\mathcal{K}_{p} \times O(\Delta x) \times O\left(\frac{\Delta r}{r}\right),
$$

since $\tilde{\mathcal{K}}(\boldsymbol{x})$ and $\frac{1}{2} \tilde{\rho}_{2}(\boldsymbol{x})\left(\boldsymbol{u}_{p}^{\mathrm{R}}\right)^{2}$ are two approximations of order $O(\Delta x) \times O\left(\frac{\Delta r}{r}\right)$ of the exact kinetic energy, at least in average over $V_{p}$. Note that the residue in $O\left(\frac{\Delta r}{r}\right)$ is due to the presence of $\tilde{\rho}_{2}(\boldsymbol{x})$-see Relation (B.6).

\section{Appendix C. Dissipative terms}

The usual scalar artificial viscosity $[62,63]$ is used here for all test cases. Introducing linear and quadratic terms in compression, it writes

$$
q_{c}=Q\left[\left\{\boldsymbol{u}_{p}\right\},\left\{\boldsymbol{x}_{p}\right\}, e_{c}\right]=-q_{1} \rho_{c} c_{c} \ell_{c} \frac{\min \left\{0, \mathrm{~d}_{\mathrm{d}} V_{c}\right\}}{V_{c}}+q_{2} \rho_{c}\left(\ell_{c} \frac{\min \left\{0, \mathrm{~d}_{1} V_{c}\right\}}{V_{c}}\right)^{2},
$$

where $\ell_{c}$ is a characteristic length and $c_{c}$ is the sound speed of cell $c$. The volume derivative is given by

$$
\mathrm{d}_{t} V_{c}=\sum_{p \in P(c)} \frac{\partial V_{c}}{\partial x_{p}} \cdot \boldsymbol{u}_{p}
$$

Inertial anti-hourglassing forces [24] derive from a per-cell dissipative potential $Q_{c}$

$$
Q_{c}=\sum_{p \in P(c)} \frac{1}{2} m_{c p}\left(\boldsymbol{u}_{p}-\overline{\boldsymbol{u}}_{c p}\right)^{2} \geq 0 .
$$

The linear velocity $\overline{\boldsymbol{u}}_{c p}$ is then computed to minimize the potential $Q_{c}$-corresponding to the per-cell kinetic energy holding by modes associated with velocities $\boldsymbol{u}_{p}-\overline{\boldsymbol{u}}_{c p}, p \in P(c)$. Hence, the linear velocity is given by

$$
\begin{array}{ll}
\boldsymbol{x}_{c}=\left(\sum_{p \in P(c)} m_{c p} \boldsymbol{x}_{p}\right) / \sum_{p \in P(c)} m_{c p}, & \boldsymbol{u}_{c}=\left(\sum_{p \in P(c)} m_{c p} \boldsymbol{u}_{p}\right) / \sum_{p \in P(c)} m_{c p}, \\
\delta \boldsymbol{x}_{c p}=\boldsymbol{x}_{p}-\boldsymbol{x}_{c}, & \delta \boldsymbol{u}_{c p}=\boldsymbol{u}_{p}-\boldsymbol{u}_{c}, \\
\mathrm{~J}_{c}=\sum_{p \in P(c)} m_{c p} \delta \boldsymbol{x}_{c p} \otimes \delta \boldsymbol{x}_{c p}, & \sum_{c}=\sum_{p \in P(c)} m_{c p} \delta \boldsymbol{u}_{c p} \otimes \delta \boldsymbol{x}_{c p} \cdot\left(\mathrm{J}_{c}\right)^{-1}, \\
\overline{\boldsymbol{u}}_{c p}=\boldsymbol{u}_{c}+\Sigma_{c} \cdot \delta \boldsymbol{x}_{c p}, & \boldsymbol{f}_{c p}=\mathcal{F}\left[\left\{\boldsymbol{u}_{p}\right\},\left\{\boldsymbol{x}_{p}\right\}, e_{c}\right]=-c_{\bar{\Phi}} \frac{c_{c}}{\ell_{c}} \frac{\partial Q_{c}}{\partial \boldsymbol{u}_{p}}=-c_{\bar{\Phi}} \frac{c_{c}}{\ell_{c}} m_{c p}\left(\boldsymbol{u}_{p}-\overline{\boldsymbol{u}}_{c p}\right) .
\end{array}
$$

Anti-hourglassing magnitude is controled by coefficient $c_{\bar{\Phi}}$ which is set to $c_{\bar{\Phi}}=1$. 


\section{References}

[1] J.M. Owen, M. Shashkov, Arbitrary-Lagrangian-Eulerian remap treatments consistent with staggered compatible total energy conserving Lagrangian methods, J. Comp. Phys. 273, 520 (2014).

[2] M. Kucharik, M. Shashkov, Conservative multi-material remap for staggered multi-material Arbitrary Lagrangian Eulerian methods, J. Comp. Phys. 258, 268 (2014).

[3] D.P. Starinshak, J.M. Owen, A subzone reconstruction algorithm for efficient staggered compatible remapping, J. Comp. Phys. 296, 263 (2015).

[4] R.B. DeBar, Fundamentals of the KRAKEN code, LLNL, Report UCIR-760 (1974).

[5] M. Wilkins, Calculation of elastic-plastic flow, p. 211, in B.J. Alder, S. Fernbach, M. Rotenberg, Methods in computational physics: advances in research and applications. Vol. 3, Fundamental methods in hydrodynamics, Academic Press, 1964.

[6] P.H. Maire, R. Abgrall, J. Breil, R. Loubère, B. Rebourcet A nominally second-order cell-centered Lagrangian scheme for simulating elastic-plastic flows on two dimensional unstructured grids, J. Comp. Phys. 235, 626 (2013).

[7] C.W. Hirt, A.A. Amsden, J.L. Cook, An Arbitrary Lagrangian-Eulerian computing method for all flow speeds, J. Comp. Phys. 135, 203 (1974).

[8] A.J. Barlow, P.H. Maire, W.J. Rider, R.N. Rieben, M.J. Shashkov, Arbitrary Lagrangian-Eulerian methods for modeling high-speed compressible multi-material flows, J. Comp. Phys. 322, 603 (2016).

[9] L.G. Margolin, Arbitrary Lagrangian-Eulerian (ALE) methods a personal perspective, LANL, Report LA-UR-13-24124 (2013).

[10] W. Boscheri, M. Dumbser, A direct Arbitrary Lagrangian-Eulerian ADER-WENO finite volume scheme on unstructured tetrahedral meshes for conservative and non-conservative hyperbolic systems in 3D, J. Comp. Phys. 275, 484 (2014).

[11] T. Vazquez-Gonzalez, A. Llor, C. Fochesato, A novel GEEC (Geometry, Energy, and Entropy Compatible) procedure applied to a staggered direct-ALE scheme for hydrodynamics, Eur. J. Mech. B/Fluid 65, 494 (2016).

[12] P. Hauret, P. Le Tallec, Conservation analysis for integration schemes in quasi-incompressible elastodynamics, École Polytechnique CMAP, Internal Report 499 (2002).

[13] P. Hauret, P. Le Tallec, Energy-controlling time integration methods for nonlinear elastodynamics and low-velocity impact, Comp. Meth. App. Mech. Eng. 195, 4783 (2006).

[14] D.E. Burton, N.R. Morgan, T.C. Carney, M. Kenamond, Reduction of dissipation in Lagrange cell-centered hydrodynamics (CCH) through corner gradient reconstruction (CGR), J. Comp. Phys. 299, 229 (2015).

[15] M. Kenamond, M. Bement, M. Shashkov, Compatible total energy conserving and symmetry preserving Arbitrary Lagrangian Eulerian hydrodynamics in 2D rz-cylindrical coordinates, J. Comp. Phys. 268, 154 (2014).

[16] D.P. Starinshak, J.M. Owen, A multi-material extension to subzonal reconstruction, J. Comp. Phys. 313 , 594 (2016).

[17] R. Loubère, M.J. Shashkov, A subcell remapping method on staggered polygonal grids for Arbitrary Lagrangian Eulerian methods, J. Comp. Phys. 209 (1), 205 (2005).

[18] D. Bailey, M. Berndt, M. Kucharik, M. Shashkov, Reduced-dissipation remapping of velocity in staggered Arbitrary Lagrangian Eulerian methods, Comput. Appl. Math. 233 (12), 3148 (2010).

[19] D. Bailey, Second-order monotonic advection in LASNEX, Laser Program Annual Report'84, Report UCRL-50021-84, 3-57 (1984).

[20] L.G. Margolin, M. Shashkov, Remapping, recovery and repair on a staggered grid, Comput. Methods Appl. Mech. Eng. 193 (39), 4139 (2004).

[21] R. Loubère, M. Staley, B. Wendroff, The repair paradigm: New algorithms and applications to compressible flow, J. Comp. Phys. 211, 385 (2006).

[22] M. Shashkov, B. Wendroff, The repair paradigm and application to conservation laws, J. Comp. Phys. 198, 265 (2004).

[23] R. Anderson, V. Dobrev, T. Kolev, R. Rieben, Monotonicity in high-order curvilinear finite element ALE remap, Int. J. Numer. Methods Fluids 77, 249 (2015).

[24] A. Claisse, P. Le Tallec, A. Llor, A. Marboeuf, Reducing mesh distortions and asymmetries in space-staggered Lagrangian hydro-schemes: variable node masses and inertial filtering, In preparation.

[25] A. Marboeuf, Schémas ALE multi-matériaux totalement conservatifs pour l'hydrodynamique, Ph.D. Thesis, Université Paris-Saclay, CEA (2018).

[26] A. Claisse, A. Llor, C. Fochesato, Further exploration of the Lagrangian CSTS (Conservative Space- and Time-Staggered) hydrodynamic scheme, Oral talk, Europ. Conf. Comput. Fluid Dynamics (ECFD VI), July 20th-25th, Barcelona, 2014.

[27] A. Claisse, A. Marboeuf, P. Le Tallec, A. Llor, Conservative axisymmetric space- and time-staggered hydrodynamic schemes, Oral talk, Int. Conf. Num. Meth. Multi-Material Fluid Flow, September 7th-11th, Wurzburg, 2015.

[28] D.E. Burton, Exact conservation of energy and momentum in staggered-grid hydrodynamics with arbitrary connectivity, Lect. Notes Phys. 395, 7 (1991)

[29] A. Llor, A. Claisse, C. Fochesato, Energy preservation and entropy in Lagrangian space- and time-staggered hydrodynamic schemes, J. Comp. Phys. 309, 324 (2016).

[30] A. Marboeuf, C. Claisse P. Le Tallec, A. Llor, Theoretical and numerical studies of entropy production in axisymmetric geometry for Lagrangian CSTS hydrodynamic schemes, Poster, Int. Conf. Num. Meth. Multi-Material Fluid Flow, September 7th-11th, poster, Wurzburg, 2015

[31] J.K. Dukowicz, J.W. Kodis, Accurate conservative remapping (rezoning) for arbitrary Lagrangian-Eulerian computations, SIAM J. Sci. Stat. Computing 8, 305 (1987).

[32] B. VanLeer, Towards the ultimate conservative difference scheme, J. Comp. Phys. 32, 101 (1979).

[33] D.L. Youngs, Time dependent multi-material flow with large fluid distortion, in: K.W. Morton, M.J. Barnes (Eds), Numerical Methods for Fluid Dynamic, Academic Press, 273 (1982).

[34] M. Kucharik, R.V. Garimella, S.P. Schofield, M.J. Shashkov, A comparative study of interface reconstruction methods for multi-material ALE simulations, J. Comp. Phys. 229, 2432 (2010). 
[35] R. Scardovelli, S. Zaleski, Interface reconstruction with least-square fit and split Eulerian-Lagrangian advection, Int. J. Numer. Methods Fluid 41, 251 (2003).

[36] S. Diot, M.M. Francois, E.D. Dendy, An interface reconstruction method based on analytical formulae for 2D planar and axisymmetric arbitrary convex cells, J. Comp. Phys. 275, 53 (2014).

[37] E. Van Renterghem, CEA, Internal report (1999).

[38] T. Vazquez-Gonzalez, A. Llor, CEA, Internal report (2013).

[39] A.V. Wilchinsky, K. Hutter, On thermodynamic consistency of turbulent closures, Theor. Comp. Fluid Dyn. 15, 23, (2001).

[40] P.L. Roe, T.B. Lung, J. Maeng, New approaches to Limiting, 22nd AIAA Comput. Fluid Dynamics Conference (2015).

[41] S.M. Bakhrakh, V.F. Spiridonov, A.A. Shanin, Method for calculating gasdynamic flows of an inhomogeneous medium in Lagrange-Euler variables, Akademiia Nauk SSSR Doklady, T. 276 (1984).

[42] D.J. Benson, Computational methods in Lagrangian and Eulerian hydrocodes, Comp. Meth. App. Mech. Eng. 99, 23 (1992).

[43] W.E. Pracht, Calculating three-dimensional fluid flows at all speeds with an Eulerian-Lagrangian computing mesh, J. Comp. Phys. 17, 132 (1975).

[44] E.J. Caramana, P.P. Whalen, Numerical preservation of symmetry properties of continuum problems, J. Comput. Phys. 141, 174 (1998).

[45] H.G. Kolsky, A method for the numerical solution of transient hydrodynamic shock problems in two space dimensions, LANL, Report LA-1867 (1955).

[46] B.-I. Jun, A modified equipotential method for grid relaxation, LLNL, Report UCRL-JC-138277 (2000).

[47] R. Liska, M. Shashkov, P. Vachal, B. Wendroff Optimization-based synchronized flux-corrected conservative interpolation (remapping) of mass and momentum for arbitrary Lagrangian-Eulerian methods, J. Comput. Phys. 229, 5 (2010).

[48] G.A. Sod, A survey of several finite difference methods for systems of nonlinear hyperbolic conservation laws, J. Comput. Phys. 27, 1 (1978).

[49] C.-W. Shu, Essentially non-oscillatory and weighted essentially non-oscillatory schemes for hyperbolic conservation laws, Advanced numerical approximation of nonlinear hyperbolic equations, 325-432, Springer, (1998).

[50] C. Hu, C.-W. Shu, Weighted Essentially Non-oscillatory Schemes on Triangular Meshes, J. Comp. Phys. 150, 1 (1999).

[51] H.C. Yee, N.D. Sandham, M.J. Djomehri, Low-Dissipative High-Order Shock-Capturing Methods Using Characteristic-Based Filters, J. Comp. Phys. 150, 1 (1999).

[52] W. Boscheri, M. Dumbser, A direct Arbitrary Lagrangian Eulerian ADER-WENO finite volume scheme on unstructured tetrahedral meshes for conservative and non-conservative hyperbolic systems in 3D, J. Comp. Phys. 275, (2014).

[53] L.I. Sedov, Similarity and dimensional methods in mechanics, Academic Press, New York, 1959.

[54] J.R. Kamm, Evaluation of the Sedov-von Neumann-Taylor blast wave solution, LANL, Report LA-UR-00-6055 (2000).

[55] J.R. Kamm, F.X. Timmes, On efficient generation of numerically robust Sedov solutions, LANL, Report LA-UR-07-2849 (2007).

[56] S. Galera, P.-H. Maire, J. Breil, A two-dimensional unstructured cell-centered multi-material ALE scheme using VOF interface reconstruction, J. Comp. Phys. 229, 16 (2010).

[57] J. Breil, Numerical methods for Lagrangian and Arbitrary-Lagrangian-Eulerian Hydrodynamic Contribution to the simulation of HighEnergy-Density-Physics Problems, Habilitation à diriger des recherches, Université de Bordeaux, (2016).

[58] J.-F. Haas, B. Sturtevant, Interaction of weak shock waves with cylindrical and spherical gas inhomogeneities, J. Fluid Mech. 181, Cambridge University Press (1987).

[59] J. Quirk, S. Karni, On the dynamics of a shock-bubble interaction, J. Fluid Mech. 318, Cambridge University Press (1996).

[60] R. Loubère, P.-H. Maire, M. Shashkov, J. Breil, S. Galera ReALE: A reconnection-based arbitrary-LagrangianEulerian method, J. Comp. Phys. 229, 12 (2010).

[61] S. Del Pino, Metric-based mesh adaptation for 2D Lagrangian compressible flows, J. Comp. Phys. 230, 5 (2011).

[62] J. von Neumann, R.D. Richtmyer, A method for the numerical calculation of hydrodynamic shocks, J. Appl. Phys. 21, 232 (1950).

[63] R. Landshoff, A numerical method for treating fluid flow in the presence of shocks, LANL, Report LA-1930 (1955).

[64] V.F. Kurapatenko, Difference Methods for Solutions of Problems of Mathematical Physics, American Mathematical Society, Providence, RI (1966).

[65] M.L. Wilkins, Use of artificial viscosity in multidimensional fluid dynamic calculations, J. Comp. Phys. 36, 281 (1980). 